"In adopting our title of the Journal of Mental Science, published by authority of the Medico-Psychological Association, we profess that we cultivate in our pages mental science of a particular kind, namely, such mental science as appertains to medical men who are engaged in the treatment of the insane. But it has been objected that the term mental science is inapplicable, and that the term mental physiology or mental pathology, or psychology, or psychiatry (a term much affected by our German brethren), would have been more correct and appropriate; and that, moreover, we do not deal in mental science, which is properly the sphere of the aspiring metaphysical intellect. If mental science is strictly synonymous with metaphysics, these objections are certainly valid; for although we do not eschew metaphysical discussion; the aim of this JOURNAL is certainly bent upon more attainable objects than the pursuit of those recondite inquiries which have occupied the most ambitious intellects from the time of I'lato to the present, with so much labour and so little result. But while we admit that metaphysics may be called one department of mental science, we maintain that mental physiology and mental pathology are also mental science under a different aspect. While metaphysics may be called speculative mental science, mental physiology and pathology, with their vast range of inquiry into insanity, education, crime, and all things which tend to preserve mental health, or to produce mental disease, are not less questions of mental science in its practical, that is in its sociological point of view. If it were not unjust to high mathematics to compare it in any way with abstruse metaphysics, it would illustrate our meaning to say that our practical mental science would fairly bear the same relation to the mental science of the metaphysicians as applied mathematics bears to the pure science. In both instances the aim of the pure science is the attainment of abstract truth; its utility, however, frequently going no further than to serve as a gymnasium for the intellect. In both instances the mixed science aims at, and, to a certain extent, attains immediate practical results of the greatest utility to the welfare of mankind; we therefore maintain that our Journal is not inaptly called the Journal of Mental Science, although the science may only attempt to deal with sociological and medical inquiries, relating either to the preservation of the health of the mind or to the amelioration or cure of its diseases; and although not soaring to the height of abstruse metaphysics, we only aim at such metaphysical knowledge as may be available to our purposes, as the mechanician uses the formularies of mathematics. This is our view of the kind of mental science which physicians engaged in the grave responsibility of caring for the mental health of their fellow-men may, in all modesty, pretend to cultivate ; and while we cannot doubt that all additions to our certain knowledge in the speculative department of the science will be great gain, the necessities of duty and of danger must ever compel us to pursue that knowledge which is to be obtained in the practical departments of science with the earnestness of real workmen. The .captain of a ship would be none the worse for being well acquainted with the higher branches of astronomical science, but it is the practical part of that science as it is applicable to navigation which he is compelled to study".-Sir J. C. Bucknill, M.D., F.R.S. (Journ. Ment. Sci., vol. vii, 1861, p. 137).

Made and Printed in Great Britain. 


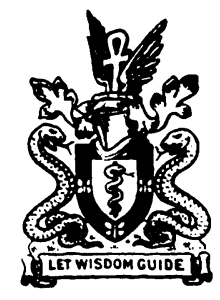

\title{
THE ROYAL MEDICO-PSYCHOLOGICAL ASSOCIATION
}

\section{YEAR BOOK \\ 1933}

\author{
CONTENTS \\ COUNCIL AND OFFICERS \\ STANDING AND SPECIAL COMMITTEES \\ TRAINING INSTITUTIONS FOR NURSES \\ HONOURS ROLL
}

MEDALS, PRIZES, AND CERTIFICATES

LIST OF MEMBERS, ETC.

\begin{abstract}
Members are particularly requested to eend notification of changes of address, honours, and additional qualifications to the Secretary, 11, Chandos Street, London, W.1; and

if they desire such to be published, to the Editors of the Journal, Cane Hill Mental Hospital, Coulsdon Surrey.
\end{abstract}


THE COUNCIL AND OFFICERS, 1932-38.

Officers.

President.-ROBERT BROWN CAMPBEll, M.D., F.R.C.P.E., J.P.

M. A. COLLINS, O.B.E., M.D. (South-Eastern).

Vice-PRESIdents AND
E. BARTON WHITE, M.R.C.S., L.R.C.P. (South-Western).

Divisional Chairmex J. H. MACDONALD, M.B., Ch.B., F.R.F.P.S.Glasg. (Scottish).

J. O'CONOR DONELAN, L.R.C.P.\&S.I., M.P.C. (Irish).

President-Elect.-FRaNk DOUGlas TURNER, M.B., M.R.C.S., L.R.C.P.

Ex-President.-RICHARD ROBERT LEEPER, L.R.C.P., F.R.C.S.I.

Treasurer.-GEORGE WILLIAM SMITH, O.B.E., M.B.

HoN. General Secretary.-REGiNALD WORTH, O.B.E., M.B.

Registrak.-DANIEL F. RAMBAUT, M.A., M.D.

Hos. Librarias.-J. R. WHITWELl, M.B.

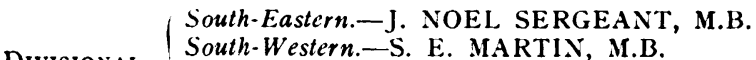

Divisioval South-Western.-S. E. MARTIN, M.B.

ISON RUSSELL, M.B., F.R.F.P.S.

Scottish.-W. M. BUCHANAN, M.B.

Parliamentary Committee.

ChatrMaN.-NATHAN RAW, C.M.G., M.D., F.R.C.S.E., F.R.S.E

Vice-Chairman.-W. F. MENZIES, B.Sc., M.D., C.M., F.R.C.P.

SeCRETARY.-H. G. L. HAYNES, M.R.C.S., L.R.C.P.

Educational Committee.

Chairman.-F. R. P. TAYloR, M.D.

Vice-ChatrmaN.-DONALD ROSS, M.B.

Secretary.-W. G. MASEFIELD, M.R.C.S., L.R.C.P.

Library Committee.

Chairma.i-J. R. WhitWEll, M.B.

SECRETARY.-C. F. F. MCDOWALL, M.D.

Research and Clinical Committee.

Chatrman.-F. L. GOlla, M.A., M.B., B.Ch., F.R.C.P.

Vice-Chatrman.-A. A. W. PETRIE, M.D., F.R.C.P., F.R.C.S.

SeCretarY.-P. K. McCOWAN, M.D., M.R.C.P., D.P.M.

The Journal of Mental Science.

(DOUGLAS McRAE, M.D., F.R.C.P.E.

M. HAMBLIN SMITH, M.A., M.D.

EDITORS ALEXANDER WALK, M.D., D.P.M.

I G. W. T. H. FLEMING, M.R.C.S., L.R.C.P., D.P.M.

Non-Official Members.

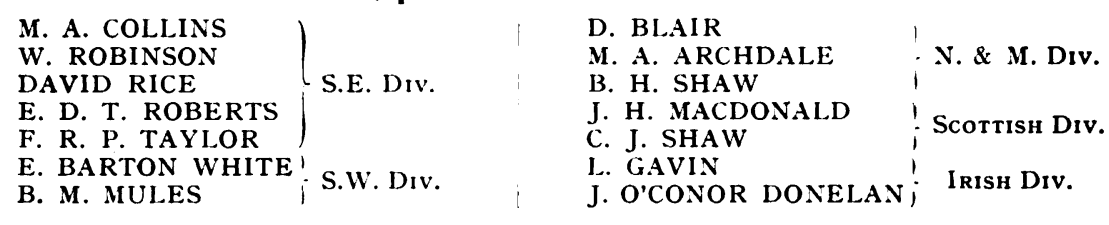

Nominated Members.

W. J. T. KIMBER, J. ERNEST NICOLE, J. J. O'REILLY, W. STARKEY, J. S. I. SKOTTOWE, AIDAN G. W. THOMSON. 
THE STANDING COMMITTEES.

[The President, Generab Secretary, Treasurer, an Editor, and the Divisional Secretaries are ex-officio Members.]

\section{Parliamentary Committee.}

D. BLAIR.

J. SHAW BOLTON.

SIR HUBERT BOND.

C. W. BOWER

A. HELEN A. BOYLE.

G. L. BRUNTON.

M. A. COLLINS.

H. DOVE CORMAC.

W. H. COUPLAND.

W. R. DAWSON, O.B.E.

J. F. DIXON

R. EAGER.

A. T. W. FORRESTER.

C. W. FORSYTH.

J. R. GILMOUR.

T. SAXTY GOOD.

H. G. L. HAYNES (Secretary).

G. W. B. JAMES.

W. BROOKS KEITH.

NEIL T. KERR.
R. L. LANGDON-DOWN.

T. C. MACKENZIE.

DOUGLAS MCRAl.

E. MAPOTHER.

HAMILTON MARR.

W. F. MENZIES.

A. W. NEILL.

(Vice-Chairman)

M. J. NOLAN.

NATHAN RAW (Chairman).

W. ROBINSON.

DONALD ROSS.

R. PERCY SMITH.

R. MACKENZIE STEWART

F. R. P. TAYLOR.

F. DOUGLAS TURNER.

E. BARTON WHITE.

Sir FREDERICK WILLIS.

H. WOLSELEY-LEWIS.

Educational Committee.

J. SHAW BOLTON.

SIR HUBER'T BOND.

A. HELEN A. BOYLE.

J. BRANDER.

M. A. COLLINS

H. DOVE CORMAC.

W. H. COUFLAND.

A. W. DANIEL.

W. R. DAWSON, O.B.E.

J. F. DIXON

R. EAGER.

A. T. W. FORRESTER.

J. R. GILMOUR.

T. SAXTY GOOD

S. J. GRAHAM.

G. H. GRILLS.

D. K. HENDERSON.

R. FITZROY JARRETT.

NEIL T. KERR.

W. J. T. KIMBER.

H. B. LEECH.

E. S. LITTELJOHN.

J. MACARTHUR.

T. C. MACKENZIE.

DOUGLAS MCRAE.

E. MAPOTHER.

W. G. MASEFIELD

W. F. MENZIES.

A. W. NEILL.

M. J. NOLAN.

F. M. RODGERS

DONALD ROSS

(Vice-Chairman)

R. PERCY SMITH.

F. R. P. TAYLOR

w. REES THOMAS

(Chairman).

F. DOUGLAS TURNER.

E. BARTON WHITE.

Also the Registrar and Examiners.

Library Committee.
A. HELEN A. BOYLE.
H. DEVINE.
G. W. T. H. FLEMING.
T. B. HYSLOP.
E. MAPOTHER.
COLIN MCDOWALL (Setretary).

W. McWILLIAM.
J. E. NICOLE.
W. H. B. STODDART.
J. R. WHITWELL

(Chairman). 


\title{
RESEARCH AND CLINICAL COMMITTEE.
}

\author{
Elected Members.
}

Central A uthoritics.-SIR HUBERT BOND, F. J. DEANE, D. L. KELLY, HAMILTON MARR. County, Borough, and Registered Mental Hospitals.-H. DEVINE, D. K. HENDERSON, W. F. MENZIES, W. D. NICOL, M. J. NOLAN, J. G. PORTER PHILLIPS. Private Mental Hospitals. - C. W. BOWER, J. N. GLAISTER, H. J. NORMAN. H.M. Prison Service.-W. NORWOOD EAST, M. HAMBLIN SMITH. Mental Deficiency Services.-E. S. LITTELJOHN, W. A. POTTS.

Consultant Psychiatrists and Neurologists.-BERNARD HART, J. E. MIDDLEMISS.

Teachers of Psychiatry.-J. SHAW BOLTON, J. O'CONOR DONELAN, E. MAPOTHER.

General Medicine.-E. BRAMWELL, A. CORSAR STURROCK.

Neurology.-A. NINIAN BRUCE, F. L. GOLLA.

Pathology.-E. GOODALL, F. A. PICKWORTH.

Psychology.-T. BEATON, W. H. B. STODDART.

Secretaries of Sub-Committees.

Treatment of Gencral Paralysis, etc.-W. D. NICOL.

Psychotherapy and Psycho-Pathology.-J. E. NICOLE.

Infectious Diseases in Mental Hospitals.-M. J. McGRATH.

Pathology, Bacteriology and Bio-Chemistry.-K. K. DRURY, F. A. PICKWORTH.

Clinical Psychiatsy.-G. W. T. H. FLEMING.

Care and Treatment of Mental Defectives.-E. O. LEWIS.

Study Tours and Post-Graduate Education.-A. E. EVANS.

- Other Representatives of Sub-Committees.

R. J. A. BERRY.

W. M. FORD-ROBERTSON.

M. E. FRANKLIN.

H. G. L. HAYNES.

DONALD ROSS.

G. DE M. RUDOLF.

Chairman.-F. L. GOL.LA.
G. E. SHAND.

B. H. SHAW.

I. D. SUTTIE.

W. REES THOMAS

H. YELLOWLEES.

Vice-Chairman.-A. A. W. PETRIE. Secretary.-P. K. McCOWAN.

SPECIAL COMMITTEES.

Mental Nursing Advisory Committee to the General Nursing Councils.

England and Wales.-H. DOVE CORMAC, W. G. MASEFIELD, GEORGE W. SMITH, F. R. P. TAYLOR, A. A. D. TOWNSEND.

Scotland.-W. M. BUCHANAN, R. D. HOTCHKIS, T. C. MACKENZIE, DOUGLAS MCRAE, DONALD ROSS

Northern Ireland.-F. J. DEANE, DOROTHY GARDNER, N. B. GRAHAM, M. J. NOLAN, J. WATSON.

Irish Free State.-S. BLAKE, J. O'CONOR DONELAN, L. GAVIN, R. R. LEEPER, J. C. MARTIN.

\section{Mental Deficiency Nursing Handbook Committee.}

C. G. A. CHISLETT, Miss R. DARWIN, W. J. T. KIMBER (Secretary), R. L. LANGDONDOWN, E. S. LITTELJOHN, A. M. MCCUTCHEON, W. A. POTTS, A. ROTHERHAM, E. B. SHERLOCK (Chairman), A. F. TREDGOLD, F. DOUGLAS TURNER. 
HONORARY AUDITORS.

\author{
G. F. BARHAM, M.D. \\ R. J. STILWELL.
}

EXAMINERS FOR THE CERTIFICATE IN PSYCHOLOGICAL MEDICINE.

ENGland.-H. DEVINE, O.B.E., M.D., F.R.C.P.; E. MAPOTHER, M.D., F.R.C.P. SCOTLAND-DOUGLAS MCRAE, M.D., F.R.C.P.E.; R. DODS BROWN, M.D., F.R.C.P.E. IRELAND-J. O'CONOR DONELAN, L.R.C.P.\&S.Irel.; M. J. NOL.A., L.R.C.P.\&S.Irel.

\title{
EXAMINERS FOR THE NURSING CERTIFICATES.
}

Mental-Preliminary: ALEXANDER DICK, M.C., M.B.; M. J. McGRATH, M.B.; W. BROOKS KEITH, M.C., M.D.: J. F. W. LEECH, M.D., DOROTHY GARDNER, M.B. Final: D. ROSS, M.B.; J. MCGARVEY, M.13. : G. L. BRUNTON, M.D. NORMAN R. PHILLIPS, M.D.; P. MORAN, M.B.

MENTAL defectives-Final: E. B. SHERLOCK, M.D.; R. D. CLARKSON, B.Sc., M.D.; G. H. KEENE, M.D.

Nurse examiners.-Miss C. M. MOORE, R.M.P.N.C., S.R.N.Eng.; Miss M. J. GOW, R.M.P.N.C., S.R.N.Scot. ; MISs DELANEY, R.M.P.N.C., S.R.N.Irel.; Miss M. E. WEBBERLEY, R.M.P.N.C., S.R.N.Eng. ; MISS D. JONES.

\section{LIST OF EXAMINATION AREAS AND AREA EXAMINATION SECRETARIES} FOR THE MENTAL NURSING CERTIFICATE.

Examination Areas.

South-EAsters Division:

(I) Northern Area (North of Thames) . . . Dr. W. J. T. KIMBER.

(2) Southern Area (South of Thames) $\quad$. . , A. A. II. PETRIE.

South-Westers Division :

(I) Bristol Area

(2) Cardiff Area

(3) Exeter Area

(4) Oxford Area

(5) Salisbury Area
Dr. E. BARTON WHITE.

, J. S. I. SKOTTOWE.

", D. MCKINLEY REID.

J. F. W. LEECH.

", C. W. S. DAVIES-JONES.

Northern and Midlaxd Division:

(I) Nottingham Area

(2) Manchester Area

(3) Leeds Area

(4) York Area

(5) Newcastle Area

(6) Birmingham Area

Scottish Division:

One Area

Dr. J. BAIN.

"H. DOVE CORMAC.

, J. R. GILMOUR.

" T. M. DAVIE.

," T. M. DAVIE. DRAKE-BROCKMAN

", c. W. FORSYTH.

Dr. A. M. DRYDEN

IRISH DIVISION :

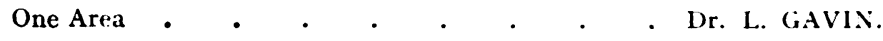


vii

\section{TRAINING INSTITUTIONS FOR NURSES.}

[For recognition as Training Schools, Mental Institutions and Institutions for Mental Defectives must be visited and reported on favourably by a referee or referees appointed by the Training Sub-Committee or Registrar.]

\section{FOR TRAINING MENTAL NURSES.}

(I) England and Wales.

(i) All County, City, and Borough Mental Hospitals, including Tooting Bec Hospital.

(ii) Criminal Asylum: Broadmoor.

(iii) Registered Hospitals: St. Andrew's (Northampton), Manchester Royal, Holloway Sanatorium, Bethlem Royal, The Retreat, Barnwood House, Bootham Park, Coton Hill, Wonford House, The Warneford, The Coppice, The Lawn (for Female Nurses only).

(iv) Special Hospitals: Netley (D Block), Roval Naval Hospital (Yarmouth), Maudsley Hospital, St. George's Retreat, Burgess Hill, Sussex (with the Hospital of St John and St. Elizabeth, for Training in Medical and Surgical Nursing).

(v) Private Mental Hospitals : The Old Manor, Camberwell House, Laverstock House, Peckham House, Brislington House, Ticehurst House, Northumberland House, The Priory, Middleton Hall, Bailbrook House, Brooke House (for Female Nurses only).

(vi) Municipal Hospitals: Erdington House (Birmingham), Smithdown Road (Liverpool).

(2) Scotland.

(i) Royal Asylums and Hospitals: Aberdeen, Crichton (Dumfries), Dundee, Edinburgh, Glasgow (Gartnavel), Montrose, James Murray's (Perth).

(ii) All City and District Asylums and Mental Hospitals.

(iii) Poor Law Asylum: Greenock.

(iv) Poor Law Hospital : Stobhill Hospital (Glasgow).

(v) Private Asylum: New Saughton Hall (Polton).

(3) Ireland.

(i) All County, City and District Asylums and Mental Hospitals.

(ii) Criminal Asylum : Dundrum.

(iii) Chartered Hospitals : St. Patrick's.

(iv) Private Asylum: Farnham House (for Female Nurses only).

(4) Federated Malay States.

Central Mental Hospital: Tanjong Rambutan, Perak.

(5) INDIA.

Ranchi Mental Hospital for Europeans, Kankè, Province of Bihar and Orissa.

FOR TRAINING THOSE NURSING MENTAL DEFECTIVES.

(I) ENgland.

(i) State Institution: Rampton.

(ii) Certified Institutions: Brentry, Calderstones, Coleshill Hall, Farmfield, Hortham (Bristol), Legbourne Grange Colony (for Female Nurses only), The Manor, Middlesex Colony (Shenley), Monyhull Colony, Royal Albert Institution, Royal Earlswood, Royal Eastern Counties, Sandhill Park (with ancillary premises), South Side (Streatham), Western Counties Institution.

(iii) Approved Institutions: Darenth, Leavesden, Caterham, Fountain (for Female Nurses only), Great Barr Park Colony.

(iv) Certified Houses : Normansfield.

(2) Scotrand.

Baldovan Institution, Royal Scottish National Institution, Stoneyetts Institution. 
viii

THE LIBRARY AND SECRETARY'S OFFICE.

II, Chandos Street, London, W. I.

Secretary and .4 ssistant Librarian.-Miss. M. HIGGINS.

PUBLICATIONS.

From Messrs. Adlard \& Son, Ltd.:

Year Book of the Association. Price 6d. Post free.

The Charter and Bye-Laws of the Association. Price $1 /-$. Post free.

Engraved Book-Plates of the Association. Price $1 / \cdot$. Post free.

Portrait of Dr. Samuel Hitch. Price I/-. Post free.

“"The Clinical Study of Mental Disorders." Price 6/.. Post free.

" First Report on the Comparative Incidence of Cancer in Mental Hospital Patients and in the General Population of England." Price 9d. Post free.

"The State Registration of Mental Nurses." Parts I and II. Price 6d. each. Post free.

"Memorandum on the Diplomas in Psychological Medicine." Price $2 d$. Postage $\frac{1}{t} d$.

"An Account of a Tour of Dutch Mental Hospitals and Clinics." Price I/-. Post free.

From Messrs. J. \& A. Churchill :

“The Journal of Mental Science." Published Quarterly. Price 7/6. Postage extra. General Index of the Journal, vol. lv to lxviii (inclusive). Price 2/6. Post free.

Binding Cases for the General Index, price 2/. (post free), are obtainable from Messrs. Adlard $\&$ Son, Ltd., who also undertake binding, including case, for $4 / 6$.

Catalogue of the Association's Library. Price 2/-. Post free.

From Messrs. Baillière, Tindall \& Cox :

"Handbook for Mental Nurses." 7th edn. 1923. Price 6/- net.

"Manual for Mental Deficiency Nurses." 1931. Price 6/- net.

From the Registrar, St. Andrew's Hospital, Northampton :

" Regulations" $\}$ for the Examinations for the Certificates of Proficiency in Mental Nursing

" Rullabuses" $\}$ and in Nursing Mental Defectives. Price 2d. each. Postage $1 d$. 
HONOURS ROLL.

Year.
1883
1884
1885
1886
1887
1888
1889
1890
1891
1891
1892
1893
1893
1894
1895
1896
1897
1898
1899
1900
1901
1902
1903
1904
1905
1906
1907
1908
1909
1910
1911
1912
1913
1914
1915
1916
1917
1918
1919
1920
1921
1922
1923
1924
1925
1926
1927
1928
1929
1930
1931
1932

Gaskell Medal and Prize.

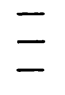

J. E. D. Mortimer.

T. B. Hyslop.

G. M. Robertson.

Nathan Raw.

G. R. Wilson.

G. W. F. Macnaughton.

W. R. Dawson.

C. Hubert Bond.

二

Maurice Craig.

W. H. B. Stoddart.

G. H. Grills.

$$
\text { - }
$$

J. M. Rutherford.

H. Devine.

J. G. P. Phillips.

W. Boyd.

W. R. Thomas.

J. C. Woods.

J. Walker.

$$
\text { - }
$$

J. T. H. Madill.

Mary Barkas.

W. S. Dawson.

G. de M. Rudolf.

Elizabeth Casson.

F. R. Martin.

Alexander Walk.

G. W. T. H. Fleming.

Muriel L. M. Northcote.
Bronze Medal and Prize.

J. Wiglesworth.*

S. R. Macphail.

T. D. Greenlees.*

J. Wiglesworth.

J. C. Mackenzie.

W. J. Smythe.

G. M. Robertson.

A. W. Campbell.

C. Beadles.

J. Turner.

J. R. Lord.

F. G. Crookshank.

C. C. Easterbrook.

A. A. D. Townsend.

C. J. Shaw.

S. C. Howard.

C. F. F. McDowall.

H. Morton.

G. H. Garnett.

D. McKinley Reid.

J. C. Wootton.

R. Eager.

W. S. Dawson.

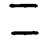

P. K. McCowan.

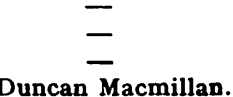

J. H. Ewen.

W. R. Ashby. 
MEDALS AND PRIZES.

The Gaskell Gold Medal and Prize.

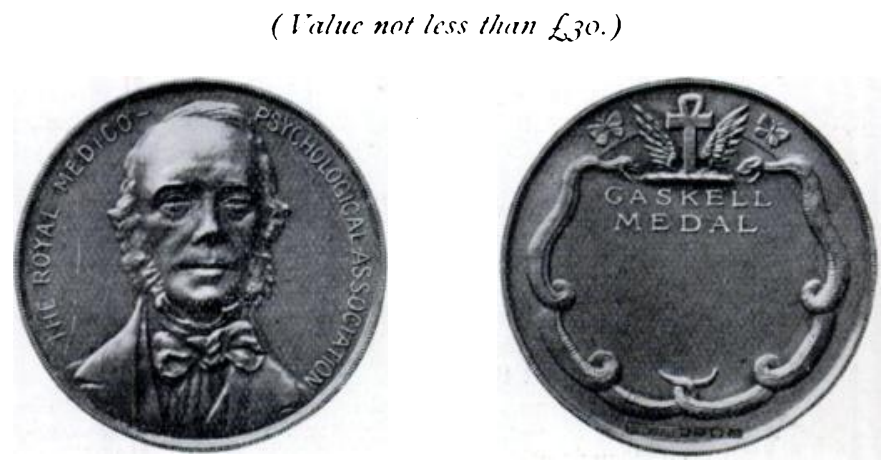

This . Medal and Prize as established, at the Annual Meting hild on fugust 9, I886, in honour of Samucl Gaskell, Esq., F.R.C.... at one time Medical superintendent of the county Asylum, Lancaster, and afteruards for s 7 years a Commissioner in Lunacy. Qpon his death on .1/arch $\mathrm{x}$ (6, 1886 , his sister, Virs. Holland, gar't 1000 to the Association, and other members of the family contributed further sums amounting to $f 340$. The interest of this sum is giv'n annually as a prize to the candidate ath" most distinguishes himself in an Lxamination in the subjects heriundir mentiencid.

The examination must he held in England according to the terms of the Trust.

(I) Candidates must produce evidence: (a) Of having attained the age of 23 . (b) Of having been qualified Medical Officers in one or more mental hospitals or clinics in psychiatry in the United Kingdom or elsewhere in the British Empire for at least two years. (c) Of possessing the Certificate in Psychological Medicine of this Association or a degree or diploma in Psychological Medicine of one of the Cniversities or Examining Boards having the power to grant medical qualifications registrable in the British lsles or elsewhere in the British Empire.

(2) Candidates will be examined in : (a) Psychology. (b) Anatomy, Histology and Physiology of the Nervous System, reproductive and endocrine glands; Pathology of the above, especially in relation to mental disorders. (c) The diagnosis, prognosis, treatment and legal relations of mental disorders. (d) Clinical cases. 
The Bronze Medal and Prize.

( linlue Ten (iminers.)
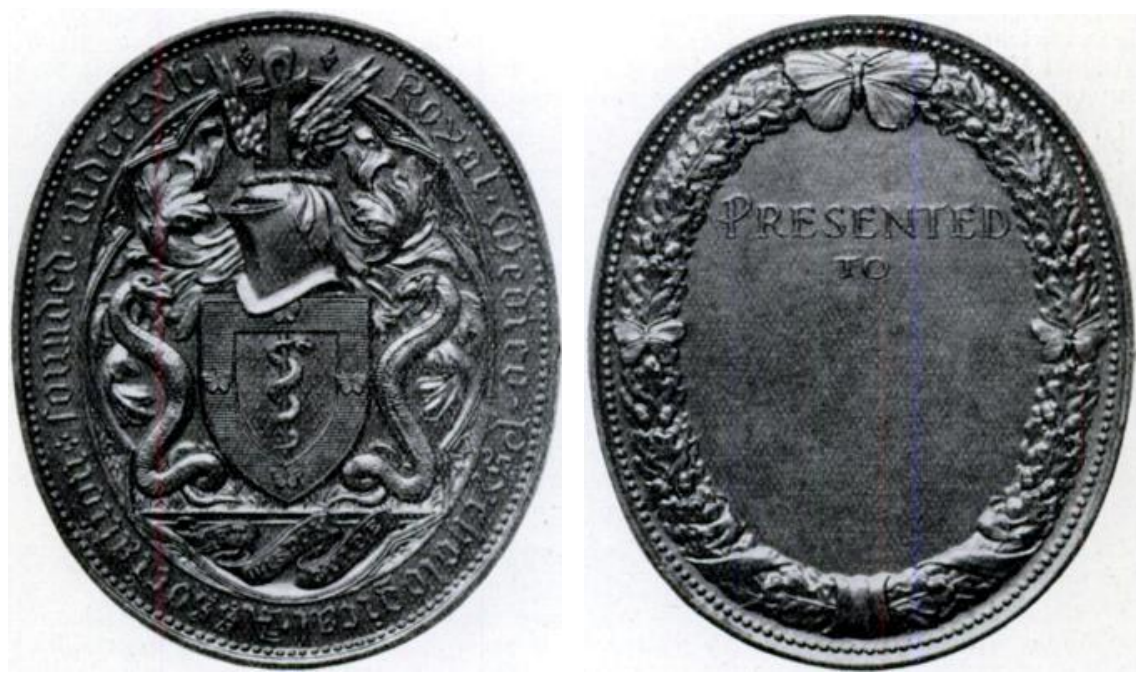

The Bronse Medal and Prise aills istablished at the Annual Mecting held on Alugust 2,1882, and is atiarded to any assistant medical officer of a mental hospital (public "r pritate) for the best dissertation on a clinical or pathological subject relating: to mintal disordirs.

The following is an abstract of the conditions :

(1) The dissertation to be written in English.

(2) Each dissertation to be distinguished by a motto or device, and accompanied by a sealed envelope containing the name and residence of the author, and having on the outside a motto or device corresponding with that on the dissertation.

(3) The dissertation shall not exceed ro,oon words in length.

(4) The dissertation and every accompanving drawing and preparation will become the property of the Association, to be published in the Journal at the discretion of the Editors.

(5) The dissertations not approved, with their accompanving drawings and preparations, will, upon application within one year, be returned, together with the sealed envelopes unopened; if not claimed within one year they will become the property of the Association.

(6) The dissertations for the year must be delivered to the Registrar before April 30.

(7) No prize will be awarded if none of the dissertations is of sufficient merit. 


\title{
Divisional Prizes for Assistant Medical Officers.
}

\author{
( Two Prizes of £ro and £5 respectively.)
}

The proposal to award Divisional Prizes under the conditions set out below emanated from the Irish Division and was adopted at the Annual Meeting held on July 13, I91 1.

(I) Two prizes of $f$ io and $f 5$ respectively will be awarded annually by the Association (provided sufficient merit be shown) for the best paper read during the preceding calendar year at a Divisional Meeting by an Assistant Medical Officer or Assistant Physician in a Psychiatric or Neurological Institution or Service. (See also Regulation No. 7.)

(2) Competitors must be members of the Association.

(3) Only one paper by a competitor can be entered during any competitive year. The same applies to a paper entered by more than one author.*

(4) Only papers certified by the Secretary of the Division (in the absence of the Secretary, the Chairman of the Meeting) to have been read at a meeting of the Division and to be eligible for this competition can be entered. Divisional Secretaries are to withhold their certificates in cases where there has occurred material alterations or additions.

(5) Papers certified as eligible for the competition shall be forwarded to the Registrar not later than April 30 of the following year. They shall be submitted to the Examiners for the Certificate in Psychological Medicine for report and then adjudicated on by the President. Publication shall not invalidate a paper for adjudication. $\dagger$

(6) The results shall be announced, and the prizes awarded, if any, by the President at the Annual Meeting in the year following the ompetitive year.

(7) Papers by more than one author can be entered and any prize awarded be divided among the authors.

(8) If in any competitive year one or two papers only are submitted for competition the Council may withhold either or both prizes; or it mav, after considering the report of the Adjudicator, and if in its opinion the paper or papers are of outstanding merit, award a first or second or both prizes, at its discretion.

\section{THE CERTIFICATE IN PSYCHOLOGICAL MEDICINE.}

This Certificate was founded at the Annual Meeting held on August 4,1885 , and the first examinations were held in 1886 .

Candidates must be at least twent $y$-one vears of age, and must produce a certificate of having at least three months' clinical experience of mental disorders, or of having attended a course of lectures on insanity and the practice of a mental hospital (where there is clinical teaching) for a like period, or they shall give such proof of experience in mental disorders as shall, in the opinion of the President, be sufficient.

The examination is held yearly, and is in three parts : ( 1 ) Written. (2) Oral. (3) Practical. Candidates must be registered under the Medical Act ( 1858$)$ before the certificate is bestowed.

The fee for the examination is $£ 33 s$., and intending candidates should communicate with the Registrar, who will supply date and place of examination.

Candidates failing in the examination may present themselves at the next or subsequent examinations on payment of a fee of $f I$ Is.

- This means that the joint authors (thuugh separately eligible) cannot enter another paper in the competitive year.

+ The original MS., certified in accordance with Regulation No. 4 , is to be submitted. Only under exceptional circumstances can a certified reprint of a published paper be accepted, but a reprint, where possible. should accompany the certified original MS. 
xiii

THE INSIGNIA OF THE NURSING CERTIFICATE.

The Certificate of Proficiency in Mental Nursing was founded at the Annual Mecting held on $\mathrm{f} u l y 24,1890$.

The Nursing Medal.

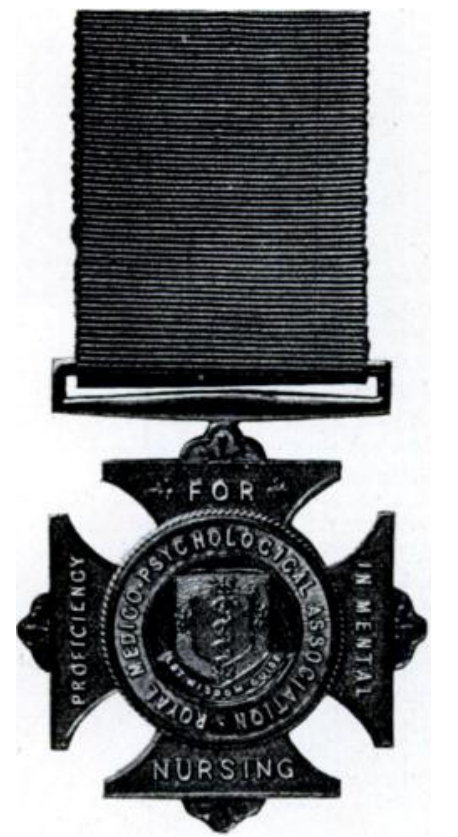

The Nursing Badge.

The Nursing Badge was fulunded at the Annual Meeting held on Fuly 11,1928 .

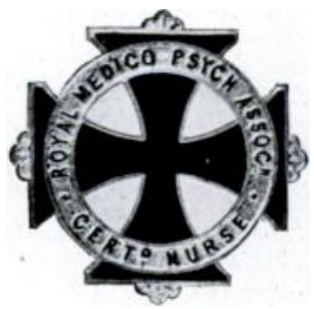

HONORARY CERTIFICATE IN MENTAL NURSING.

1928. H.R.H. The Princrss Royal. 


\section{LIST OF CHAIRMEN.}

1841. Dr. Blake, Nottingham General Asvlum.

1842. Dr. de Vitré, Lancaster Asylum.

1843. Dr. Conolly, Hanwell Asylum.

1844. Dr. Thurnam, The Retreat.

1847. Dr. Wintle, Warneford House.

1848. Willian Ley, M.R.C.S., Littlemore Asylum.

I851. Dr. Conolly, Hanwell Asylum.

I 852. Dr. Wintle, Warneford House.

LIST OF PRESIDENTS.

I 854. A. J. Sutherland, M.D., St. Luke's Hospital, London

I 855. J. Thurnam, M.D., Wilts County Asylum.

1856. J. Hitchman, M.D., Derby County Asylum.

1857. Forbes Winslow, M.D., Sussex House, Hammersmith

I 858. John Conolly, M.D., Hanwell Asylum.

1859. Sir Charles Hastings, D.C.L., Worcester.

1860. J. C. Bucknill, M.D., Devon County Asylum.

1861. Joseph Lalor, M.D., Richmond Asylum, Dublin.

1862. John Kirkman, M.D., Suffolk County Asylum.

1863. David Skae, M.D., Roval Asylum, Edinburgh.

1864. Henry Munro, M.D., Brook House.

1865. Wm. Wood, M.D., Kensington House.

866. W. A. F. Browne, M.D., Commissioner in Lunacy for Scotland.

1867. C. A. Lockhart Robertson, M.D., Haywards Heath Asvlum.

1868. W. H. O. Sankey, M.D., Sandywell Park, Cheltenham.

1869. T. Laycock, M.D., Edinburgh.

1870. Robert Boyd, M.D., Wells Asvlum.

I 871 . Henry Maudsley, M.D., The Lawn, Hanwell.

1872. Sir James Coxe, M.D., Commissioner in Lunacy for Scotland.

1873. Harrington Tuke, M.D., Chiswick House.

1874. T. L. Rogers, M.D., Rainhill Asylum.

1875. J. F. Duncan, M.D., Dublin.

1876. W. H. Parsey, II.D., Warwick County Asylum.

1877. G. Fielding Blandford, M.D., London.

1878. Sir J. Crichton-Browne, M.D., Lord Chancellor's Visitor in Lunacy.

I 879. J. A. Lush, M.D., Fisherton House.

1880. G. W. Mould, M.R.C.S., Royal Asylum, Cheadle.

881. D. Hack Tuke, M.D., London.

1882. Sir W. T. Gairdner, M.D., Glasgow.

1883. W. Orange, M.D., State Criminal Lunatic Asylum, Broadmoor.

1884. Henry Rayner, M.D., Hanwell Asylum.

1885. J. A. Eames, M.D., District Asvlum, Cork.

1886. Sir Geo. H. Savage, M.D., Bethlem Royal Hospital.

1887. Sir Fred. Needham, M.D., Barnwood House.

888. Sir T. S. Clouston, M.D., Royal Asylum, Edinburgh.

1889. H. Haves Newington, F.R.C.P., Ticehurst House.

1890. David Yellowlees, M.D., Royal Asylum, Glasgow

891. E. B. Whitcombe, M.R.C.S., City Asylum, Birmingham.

1892. Robert Baker, M.D., The Retreat.

1 893. J. Murray Lindsay, M.D., Derby County Asylum.

1894. Conolly Norman, F.R.C.P.I., Richmond Asylum, Dublin.

895. David Nicolson, C.B., M.D., State Criminal Lunatic Asylum, Broadmoor.

1 896. William Julius Mickle, M.D., Grove Hall.

1 897. Thomas W. McDowall, M.D., Northumberland Asylum.

1898. A. R. Urquhart, M.D., James Murray's Royal Asylum, Perth.

1899. J. B. Spence, O.B.E., M.D., Burntwood Asylum, Lichfield.

1900. Fletcher Beach, M.B., London.

r 9o1. Oscar T. Woods, M.D., District Asylum, Cork.

I902. J. Wiglesworth, M.D., Rainhill Asylum. 
1903. Ernest W. White, C.B.E., M.B., City of London Asylum, Stone.

1904. R. Percy Smith, M.D., London.

1905. T. Outterson Wood, M.D., London.

1906. Sir Robert Armstrong-Jones, C.B.E., M.D., Claybury Asylum.

1907. P. W. MacDonald, M.D., Dorchester County Asylum.

1908. Chas. A. Mercier, M.D., Flower House.

1909. W. Bevan-Lewis, M.Sc., West Riding Asylum, Wakefield.

19 10. Sir John Macpherson, M.D., Commissioner in Lunacy for Scotland.

I911. Wm. R. Dawson, O.B.E., M.D., Inspector of Lunatic Asylums, Dublin.

1912. J. Greig Soutar, M.B., Barnwood House.

1913. James Chambers, M.D., The Priory.

1914-18. David G. Thomson, C.B.E., M.D., Norfolk County Asylum.

19 18. John Keay, C.B.E., M.D., Bangour Village.

1919. Bedford Pierce, M.D., The Retreat.

1920. William F. Menzies, M.D., Cheddleton Mental Hospital.

1921. Sir Hubert Bond, K.B.E., M.D., Commissioner, Board of Control.

1922. G. M. Robertson, M.D., Royal Mental Hospital, Morningside, Edinburgh.

1923. Edwin Goodall, C.B.E., M.D., Cardiff City Mental Hospital.

1924. Michael J. Nolan, L.R.C.P.\&S.Irel., Downpatrick Asylum.

1925. Sir Frederick W. Mott, K.B.E., M.D., London.

1926. John R. Lord, C.B.E., M.D., Horton Mental Hospital, Epsom.

1927. Hamilton Marr, M.D., Commissioner, General Board of Control for Scotland.

1928. J. Shaw Bolton, D.Sc., M.D., West Riding Mental Hospital, Wakefield.

1929. Nathan Raw, C.M.G., M.D., F.R.C.S.E., F.R.S.E., Lord Chancellor's Visitor in Lunacy.

1930. T. Saxty Good, O.B.E., M.A., M.R.C.S., L.R.C.P., Oxford County and City Mental Hospital, Littlemore.

1931. R. R. Leeper, L.R.C.P.I., F.R.C.S.I., St. Patrick's Hospital, Dublin.

1932. R. B. Campbell, M.D., F.R.C.P.E., Stirling District Mental Hospital, Larbert.

\section{LIST OF MAUDSLEY LECTURERS.}

1920. Sir J. Crichton-Browne, I.L.D., D.Sc., M.D., F.R.S.

1921. Sir F. W. Mott, K.B.E., LL.D., M.D., F.R.C.P., F.R.S.

1922. Sir M. Craig, C.B.E., M.A., M.D., F.R.C.P.

1923. Charles Kirk Clarke, LL.D., M.D.

r924. J. Carswell, F.R.F.P.S.

1925. Joseph Shaw Bolton, D.Sc., M.D., F.R.C.P.

1926. George M. Robertson, M.D., F.R.C.P.E., F.R.C.S.E.

1927. Edwin Goodall, C.B.E., M.D., F.R.C.P.

1928. Sir John Macpherson, C.B., M.D., F.R.C.P.E.

1929. Charles E. Spearman, Ph.D., F.R.S.

1930. Sir Henry Alfred McCardie.

1931. Sir Hubert Bond, K.B.E., I.L.B., D.Sc., M.D., F.R.C.P.

1932. Sir Edward Farquhar Buzzard, Bart., K.C.V.O., M.A., M.D., F.R.C.P.

1933. Adolf Meyer, M.D. 


\section{HONORARY MEMBERS.}

1932. Armstrong-Jones, Sir Robert, C.B.E., D.Sc., M.D., B.S., F.R.C.P., F.R.C.S., F.S.A. D.L., J.P., 9, Bramham Gardens, London, S.W. 5 (and Plas Dinas, Carnarvon, North Wales). (Gresham Prof. of Phỵsic.) (Ord. Mem. since 1882; Gen. Sec., I 897-1906; PRESIDENT, 1906-7.)

1928. Bleuler, Eugen, Prof. Dr., Zollikerstrasse 98, Zollikon bei Zürich, Switzerland.

1900. Blumer, G. Alder, M.D., L.R.C.P.Edin., Superintendent-Emeritus, Butler Hospital ; 196, Blackstone Boulevard, Providence, R.I., L.S.A. (Ord. Mem., I 89o.)

1928. Bond, Sir Hubert, K.B.E., D.Sc., M.D.Edin., L.L.B.Lond., F.R.C.P., M.P.C., Senior Commissioner, Board of Control, Caxton House West, Tothill Street, S.W. r. (Emeritus Lect. on Psychiat., Middlx. Hosp.) (Ord. Mem., I892; General Siccretary, 1906-12; PRESIDENT, 1921-22.)

1900. Bresler, Johannes, Sanitätsrat Dr., Director of the Provincial Mental Hospital, Kreuzburg, Oberschlesien, Germany; Editor, Psychiatrisch-neurologische Wochenschrift. (Corr. Mem., 1896.)

1932. Briggs, L. Vernon, M.D., 64, Beacon Street, Boston, Mass., U.S.A. (Corr. Mem. since 1923.)

1902. Brush, Edward N., M.D., Superintendent-Emeritus, Sheppard and Enoch Pratt Hospital, Towson, Maryland; 2012, Greenberry Road, Mount Washington, Baltimore, II., U.S.A.

1925. Chamberlain, Rt. Hon. Arthur Neville, M.P., 37, Eaton Square, London, S.W. I.

1931. Chambers, James, M.A., M.D., "Estia”, Highdown Road, Roehampton, S.W. I5. (Ord. Mem. since I888; Asst. Editor, 1900-05, and Co-Editor of Journal, I905-14; PRESIDENT, I9I3-14; Treasurer, I9I7-3I.)

I909. Collins, Sir Wm. Job, K.C.V.O., B.Sc., M.D., M.S., F.R.C.S., I, Albert Terrace, Regent's

Park, London, N.W. 1.
rg12. Considine, Thomas Ivory, L.R.C.P., F.R.C.S.Irel., Medical Superintendent, Central Criminal Asvlum, Dundrum, co. Dublin.

1876. Crichton-Browne, Sir J., LL.D., D.Sc., M.D.Edin., F.R.S., 45, Hans Place, London, S.W. I. (Ord. Mem., I863; President, 1878.)

1930. Cushing, Harvey, C.B., A.M., LL.D., Sc.D., Litt.D., M.D., M.Ch., F.R.C.S., Professor of Surgery, Harvard University, Cambridge, Mass., U.S.A.

1924. Dawson, Lt.-Col. W. R., O.B.E., B.A., M.D., B.Ch.Dubl., F.R.C.P.Irel., D.P.H., M.P.C., 18, Brock Street, Bath. (Ord. Mem., I894; President, 1911-12; Co-Editor of Journal, 1920-21.)

1923. Ellis, Henry Havelock, L.S.A., 24, Holmdene Avenue, Herne Hill, S.E. 24.

1922. l'Hermitte, Dr. Jacques Jean, Médecin de l'Hospice Paul Brousse, Paris ; 9, rue Marbeuf, Paris (VIII ${ }^{*}$ ), France.

1930. Janet, Pierre, M.D., Member of the Institute of France; Professor of Psychology, College of France; 54, Rue de Varenne, Paris (VIIe).

1930. von Jauregg, Hofrat Prof. Julius R. Wagner, M.D., late Professor of Psychiatry, University of Vienna ; I, Landesgerichtstr. I8, Vienna.

1924. McDougall, William, LL.D., M.B., F.R.S., Professor of Psychology in Duke University, Durham, North Carolina, U.S.A.

1932. McDowall, Thomas W., M.D., L.R.C.S., "Burwood”, Wadhurst, Sussex. (Ord. Mem. since 1870; PrESIDENT, 1897-8.)

1928. Macmillan, Rt. Hon. Lord, M.A., LL.D., Lord of Appeal in Ordinary ; 44, Grosvenor Road, Westminster, S.W. I.

1910. Macpherson, Sir John, C.B., M.D., C.M., F.R.C.P.Edin., “ Hillside", Cleeve, nr. Bristol. (Ord. Mem., I 886 ; President, I9Io-Ir.) 
1921. Maudsley, Sir Henry Carr, K.C.M.G., C.B.E., M.D., B.S., F.R.C.P., Consulting Physician, Melbourne Hospital, Victoria ; 8, Collins Street, Melbourne.

1926. Meyer, Adolf, M.D., Psychiatrist in Chief, Phipps Psychiatric Clinic, Johns Hopkins Hospital, Baltimore, Md., U.S.A.

193I. Nolan, Michael James, L.R.C.P.I., L.R.C.S.I., J.P., Consulting Visitor-in-Lunacy to the Lord Chief Justice of Northern Ireland, and to the Chief Justice, Irish Free State : Medical Superintendent, Down County Mental Hospital, Downpatrick. (Ord. Mem. since 1888 ; President, I924-5.)

1922. Pactet, Dr. Prançois Florentin, Médecin en chef de l'Asile de Villejuif ; 94, Avenue de la République, Villejuif, Seine, France.

1929. Pavlov, Ivan Petrovitch, M.D., Director of the Physiological Laboratories, Institute of Experimental Medicine and Academy of Science, Leningrad.

1927. Rolleston, Sir Humphry Davy, Bt., K.C.B., D.C.L., L.L.D., D.Sc., M.D., F.R.C.P., Physician Extraordinary to H.M. the King; "Martins", Scotlands Lane, Hasle. mere, Surrey.

1923. Rose, Lt.-Col. Sir Arthur, D.S.O., Chairman, General Board of Control for Scotland, 25, Palmerston Place, Edinburgh.

1924. Sandhurst, Rt. Hon. Lord, Barrister-at-Law ; Lord Chancellor's Visitor in Lunacy; Edgebrook, Sheringham, Norfolk.

Igrr. Semelaigne, Dr. Réné, 59, Boulevard de Montmorency, Paris (XVI $\left.{ }^{\natural}\right)$, France. (Corr. Mem., 1893.)

1929. Sherrington, Sir Charles Scott, O.M., G.B.E., M.A., LL.D., D.Sc., M.D., F.R.C.P., F.R.C.S., F.R.S., Waynflete Professor of Physiology, Oxford ; 9, Chadlington Road, Oxford.

1927. Smith, Robert Percy, M.D., B.S.Lond., F.R.C.P., 42, Albion Street, London, W. 2. (Ord. Mem., I885; General Secretary, I896-97; Chairman, Educational Committee, 1899-1903; President, 1904-05.)

1929. Tanzi, Eugenio, M.D., Professor of Psychiatry, Direttore, Clinica Psichiatrica di S. Salvi, Florence, I taly:

rgor. Toulouse, Dr. Edouard, Médecin des Asiles de la Seine ; r, Rue Cabanis, Paris (XIVe), France.

1923. Willis, Sir Frederick James, K.B.E., C.B., Church House, Bramley, Guildford.

1926. Winkler, C., M.D., 35, Heerenstraat, Utrecht, Holland. (Corr. Mem., 1924.) 


\section{CORRESPONDING MEMBERS.}

1928. Bliss, Malcolm Andrews, M.D., Consultant in Neuro-Psychiatry, St. Luke's Hospital ; 3or, Himboldt Buildings, St. Louis, Minn., U.S.A.

1911. Boedeker, Geheimer Sanitätsrat, Prof. Justus Karl Edmund, Director, Zehlenhof Asylum ; Margaretenstr. 8, Berlin.

1929. Bouman, K. Herman, M.D., Professor of Psychiatry and Director of the University Psychiatric Clinic, Jan Luykenstraat 24, Amsterdam.

1929. Buscaino, V. M., M.D., Professor of Psychiatry, University of Catania, Sicily.

1897. Buschan, Sanitätsrat Dr. med. et phil. G., Friedrich Carlstrasse 7, Stettin, Germany.

1930. Campbell, Charles Macfie, M.D., Professor of Psychiatry, Harvard Medical School ; Director, Boston Psychopathic Hospital ; 74, Fenwood Road, Boston, Massachusetts, U.S.A.

1927. Charpentier, René, M.D., Secrétaire Général de la Société Medico-Psychologique de Paris; I 19, rue Perrouet, Neuilly s/Seine (Seine), France.

1904. Coroleŭ, Wilfrid, Medico forense del distrito de la Barceloneta, Aribau, 31, Barcelona, Spain.

1924. Cotton, Henry A., A.M., M.D., Medical Director Emeritus and Director of Research, State Hospital, Trenton, New Jersey, U.S.A.

r896. Cowan, F. M., M.D., ro9, Perponcherstraat, The Hague, Holland.

1929. Ebaugh, Franklin G., A.B., M.D., Professor of Psychiatry, University of Colorado; Director, Colorado Psychiatric Hospital ; 4200, East Ninth Avenue, Denver, Colorado, U.S.A.

1930. Evensen, Dr. Hans, Medical Director, Gaustad State Hospital, Vinderen, Norway ; Commissioner in Legal Medicine for Norway; President, Norwegian Psychiatric Society.

I9I I. Falkenberg, Sanitätsrat, Dr. Wilhelm, Berlin-Friedenau, Ringstr. 5

1907. Ferrari, Giulio Cesare, M.D., Director, Manicomio Provinciale, Imola, Bologna, Italy.

1930. Flournoy, Dr. Henri, 6, Rue de Mounetier, Geneva, Switzerland.

1911. Friedländer, Prof. Dr. Adolf Albrecht, Haus Sonnblick, Littenweiler, bei Freiburg i/Baden, Germany.

1931. Genil-Perrin, Dr., Chief Physician, Seine Department Mental Hospitals; General Secretary, French League for Mental Hygiene ; 99, Avenue de la Bourdonnais, Paris.

19or. Gommès, Dr. Marcel, 5, Rue Parrot, Paris (XII*).

1930. Haskovec, Prof. Dr. Ladislav, Professor of Nervous Diseases in the Charles University, Prague, Czecho-Slovakia.

1931. Hincks, Clarence Meredith, A.B., M.D., General Director of the National Committee for Mental Hygiene, 450, Seventh Avenue, New York, U.S.A.

193I. Jouchtchenko, Prof. A. J., Director, Ukrainian Government Institute of Clinical Psychiatry and Social Mental Hygiene, Kharkov, Ukraine, U.S.S.R., Konuchennaia 34.

1928. Kappers, C. U., Ariëns, M.D., Director, Netherlands Central Institution for Brain Research, Amsterdam.

1928. Kirby, George H., LL.D., M.D., Professor of Psychiatry, Columbia University ; Director, Psychiatric Institute, New York City, U.S.A.

1932. May, James V., M.D., Medical Superintendent, Boston State Hospital, Dorchester Center Station, Boston, Mass., U.S.A.

1930. Miyake, Dr. Koichi, Professor of Psychiatry, Tokio Imperial University; Director, Tokio Municipal Matsuzawa Psychopathic Hospital, Japan.

1909. Moreira, Prof. Dr. Juliano, Directeur General de l'Assistance aux Aliénés; Praia da Saudade 288 , Rio de Janeiro, Brazil.

1931. Moreno, Dr. Samuel Ramirez, Director, Manicomio General, Mexico City.

1922. Morowoka, Dr. T., Owada, I02, Shibuya, Tokyo, Japan. 
1929. Pameijer, J. H., M.D., Bildersstraat 22, The Hague.

1928. Pighini, Giacomo, M.D., Professor of Psychiatry and Director, Laboratori Scientifici, Instituto Psichiatrico di S. Lazzaro, S. Maurizio, Reggio Emilia, Via De Amicis ro, Italy.

1909. Pilcz, Prof. Dr. Alexander, VIII/2 Alserstrasse 43, Vienna, Austria.

1929. Pratt, G. K., M.D., Assistant Medical Director, National Committee for Mental Hygiene : 370, Seventh Avenue, New York, U.S.A.

1931. Riedel, Dr. Gustavo, Director, Hospital-Psychopathic Colony of Engenbo de Dentro, Rio de Janeiro, Brazil ; Hon. President, Brazilian League for Mental Hygiene ; VicePresident, Brazilian Society of Psychiatry.

1931. Roemer, Dr. med. Hans, Director, Institute for Mental Diseases, Illenau, Baden, Germany; Secretary, German Association for Mental Hygiene; Secretary, Health Welfare Committee, Baden.

1931. Ruggles, Dr. Arthur H., Medical Superintendent, Butler Hospital, Providence, R.I., U.S.A.; Lecturer in Psychiatry, Yale University, New Haven ; Chairman, Executive Committee of the National Committee for Mental Hygiene.

1922. Sano, Dr., Directeur de la Colonie de Gheel, Belgium.

1929. Scheer, W. M. van der, M.D., Psychiatrisch-Neurologische Kliniek, Groningen, Holland.

1930. Strecker, Edward A., M.D., Professor of Nervous and Mental Diseases, Jefferson Medical College; II , North Forty-ninth Street, Philadelphia, U.S.A.

1929. Stuurman, F. J., M.D., Secretary, Dutch Association for Psychiatry and Neurology ; Oegstgeest, near Leyden, Holland.

1927. Targowla, René, M.D., Chev. Leg. Hon., I69, Rue de L'Université, Paris (VIIe).

1929. Thom, Douglas A., M.D., Professor of Mental Hygiene, Tuft Medical School; 520, Conmonwealth Avenue, Boston, Mass., U.S.A.

1930. Weygandt, Dr. phil. et med. Wilhelm, Professor of Psychiatry and Director, Psychiatric Clinic, University of Hamburg ; Friedrichsbergerstr. 6o, Hamburg.

1929. Wiersma, E. D., Professor of Psychiatry and Director of the University Psychiatric Clinic, Groningen Holland.

1930. Williams, Frankwood E., D.Sc., M.D.; Editor, Mental Hygiene and Mental Hygiene Bulletin ; 44, West 12 th Street, New York City.

1928. Wimmer, August, M.D., Professor of Psychiatry, University of Copenhagen, Denmark. 


\section{ORDINARY MEMBERS OF THE ASSOCIATION.}

Alphabetical List of Ordinary Members of the Association on January 1, 1933, with the year in which they joined.

1928. Abd-el-Hakeem, Mohammed, M.B., B.Ch.Cairo, D.P.M., Assistant Medical Officer, Mental Hospital, Khanka, Egvpt.

ı9 1о. Adam, George Henry, M.R.C.S., L.R.C.P.Lond., Resident Physician, Malling Place, West Malling, Kent.

1931. Adamson, James Weeden Woodhams, M.D.Durh., M.R.C.S., L.R.C.P.Lond., L.S.A., Commissioner of the Board of Control (England and Wales); 14, Norfolk Square, Hyde Park, W. 2.

r919. Adey, J. K., M.B., C.M.Melb., Receiving House, Rọ̣al Park, Melbourne, Victoria, Australia.

1886. Agar, Samuel Hollingsworth, jun., B.A.Camb., L.S.A., M.R.C.S.Eng., Hurst House, Henley-in-Arden.

1923. Ahern, John Maurice, M.B., B.Ch.R.U.I., L.R.C.P.\&.S.Irel., Medical Officer, H.M. Prison, Parkhurst, I.W.

1923. Ainsworth, Cyrus Gerald, M.A., LL.B., M.B., B.Ch.Camb., M.R.C.S., L.R.C.P.Lond., Medical Superintendent, Graylingwell Mental Hospital, Chichester.

1931. Allan, Samuel Miller, M.B., Ch.B.Glasg., Assistant Medical Officer, Lancashire County Mental Hospital, Whittingham, Preston.

1932. Allen, James Stewart, M.B., B.Ch., B.A.O.Belf., Assistant Medical Officer, Antrim Mental Hospital, Northern Ireland.

1926. Albiston, Norman Arthur, M.B., B.S.Sỵd., D.P.M., 32, Darling Road, East Malvern, Victoria, Australia.

1924. Alexander, Douglas Reid, M.R.C.S., L.R.C.P.Lond., D.P.M., Deputy Medical Superintendent, Colney Hatch Mental Hospital, London, N. I r.

1922. Alexander, Marion Cameron, M.B., B.Ch., B.A.O.Belf., Dipl. Psych. Edin., Assistant Medical Officer, Storthes Hall Mental Hospital, Kirkburton, near Huddersfield.

1899. Allman, Dorah Elizabeth, M.B., B.Ch.R.U.I., Assistant Medical Officer, County Mental Hospital, Armagh.

1929. Anderson, John Colquhoun, B.Sc., M.B., Ch.B.Glasg., Dipl. Psych., 2, Foremount Terrace, Highburgh Road, Glasgow, W. 2.

1898. Anderson, John Sewell, M.R.C.S., L.R.C.P.Lond., Medical Superintendent, City Mental Hospital, Willerby, Hull.

1918. Anderson, William Kirkpatrick, M.D., Ch.B., F.R.F.P.S.Glasg., 2, Woodside Crescent, Glasgow. (Lect. on Ment. Dis., Andr. Coll., Glasg.)

1912. Annandale, James Scott, M.B., Ch.B.Aberd., D.P.M., Medical Superintendent, Kingseat Mental Hospital, Newmachar, Aberdeen.

1904. Archdale, Mervyn Alex., M.B., B.S.Durh., D.P.M., Medical Superintendent, Sunderland Mental Hospital, Ryhope.

1905. Archdall, Mervyn Thomas, L.S.A., L.R.C.P.\&.S.Edin., L.R.F.P.S.Glasg., St. Denys, New Milton, Hants.

1930. Armitage, Bernard William Francis, M.A., M.R.C.S., L.R.C.P., Assistant Medical Officer, Croydon Mental Hospital, Upper Warlingham, Surrey.

1930. Armstrong, Robert William, B.Sc., M.D., B.Ch.Belf., D.P.M., Deputy Medical Superintendent, Ewell Mental Hospital, Epsom, Surrey:

1930. Ashby, William Ross, B.A., M.B., B.Ch.Cantab., M.R.C.S., L.R.C.P., D.P.M., Assistant Medical Officer, Leavesden Mental Hospital; "Merrow Down", King's Langley, Herts.

1927. Atkin, Isaac, M.D., M.R.C.S., L.R.C.P.Lond., D.P.M., Assistant Medical Officer, Park Prewett Mental Hospital, Basingstoke, Hants.

r9 1o. Auden, George Augustus, M.A., M.D., B.Ch.Camb., Ph.D.Birm., F.R.C.P.Lond., D.P.H., F.S.A., School Medical Officer, Education Office, Council House, Margaret Street, Birmingham.

1891. Aveline, Henry Talbot Sydney, M.D.Durb., M.R.C.S., L.R.C.P.Lond., M.P.C., Medical Superintendent, Somerset and Bath County Mental Hospital, Cotford, near Taunton. (Secretary, S.W. Division, r905-1 I.) 
1922. Back, Frederick, M.B., B.S.Lond., M.R.C.S., L.R.C.P.Lond., D.P.M., Deputy Medical Superintendent, Sunderland Mental Hospital, Ryhope.

1926. Bailey, Josiah Reginald, M..B., Ch. B.Glasg., Medical Superintendent, Gogarburn Certified Institution, Corstorphine, Edinburgh.

1909. Bain, John, M.A., M.B., Ch.B.Glasg., Medical Superintendent, Derby Borough Mental Hospital, Rowditch.

191 3. Bainbridge, Charles Frederick, M.B., Ch.B.Edin., Deputy Medical Superintendent, Devon County Mental Hospital, Exminster.

1906. Baird, Harvey, M.D., Ch.B.Edin., Medical Superintendent, Periteau House, Winchelsea, Sussex.

1931. Baker, John Cotter, M.B., B.Ch., Medical Superintendent, Stretton House, Church Stretton, Salop.

1922. Banbury, Percy, M.R.C.S., L.R.C.P.Lond., D.P.M., Medical Superintendent, Borough Mental Hospital, Ipswich.

1930. Barber, Leonard, M.B., B.S.Durh., L.R.C.P.\&S.Edin., L.R.F.P.\&S.Glasg., D.P.M. Assistant Medical Officer, City Mental Hospital, Fishponds, Bristol.

1922. Barclay, Rachel Mary, M.A., LL.B., M.D.Edin., Dipl. Psych., 2, W. Crosscauseway, Edinburgh.

1904. Barham, Guv Foster, M.A., M.D., B.Ch.Camb., M.R.C.S., L.R.C.P.Lond., Medical Superintendent, Claybury Mental Hospital, Woodford Bridge, Essex.

1919. Barkas, Mary Rushton, Mi.Sc.X.Z., M.D., B.S., M.R.C.S., I.R.C.P.Lond., D.P.M., Medical Superintendent, The Lawn, Lincoln.

1927. Barkas, Thomas Cook, O.B.E., M.B., B.S.Durh., Resident Medical Officer, Middleton Hall, Middleton St. George, co. Durham.

1923. Barnes, Francis Gregory Lawson, M.B., B.S., M.R.C.S., L.R.C.P.Lond., D.P.M., Deputy Medical Superintendent, Horton Mental Hospital, Epsom.

1910. Bartlett, George Norton, M.B., B.S., M.R.C.S., L.R.C.P.Lond., Gosford House, Kidlington, nr. Oxford. (Secretary, S.W. Division, 1916-22.)

1932. Bates, Ralph Marshall, F.R.C.S.Eng., L.R.C.P.Lond., Resident Medical Officer, Stoke Park Colony ; Parrdown House, Stapleton, Bristol.

1902. Baugh, Leonard Dieckmann Hamilton, M.B., Ch.B.Edin., The Pleasaunce, York.

1921. Beaton, Thomas, O.B.E., M.D., B.S., F.R.C.P.Lond., Medical Superintendent, City Mental Hospital, Milton, Portsmouth. (Lect. on Ment. Dis., Bethlem Royal Hosp.) (Assistant Editor, 1926-27, and Co-Editor of Journal, 1927-31.)

1932. Beccle, Harold Charles, M. B., B.S., M. R.C.P., M.R.C.S., D.P.M., Assistant Medical Officer, kent County Mental Hospital, Maidstone.

1913. Bedford, Percy William Page, M.D., Ch.B.Edin., Dipl. Psych., Medical Superintendent, Dorset County Mental Hospital, Herrison, near Dorchester.

1922. Bell, Andrew Allan, M.B., Ch.B., F.R.F.P.S.Glasg., D.P.M., Pathologist and Assistant Medical Officer, Govan District Asvlum, Hawkhead, Glasgow.

193x. Bell, George Macdonald, M.B., Ch.B.Edin., Medical Superintendent, Ladỵsbridge Mental Hospital, Banff.

1931. Bennett, Edward Armstrong, M.C., M.A., M.D., D.P.M., Physician, The Institute of Medical Psychology, London; 97, Harley Street, W. 1.

1914. Bennett, James Wodderspoon, M.R.C.S., L.R.C.P.Lond., "Marsden", Babbacombe Road, Torquay, S. Devon.

1914. Benson, John Robinson, F.R.C.S.Eng., L.R.C.P.Lond., Resident Physician, Fiddington House, Market Lavington, Wilts, and Laverstock House, Salisbury.

1899. Beresford, Edwyn Henry, M.R.C.S., L.R.C.P.Lond., 20, Campion Road, Putney, London, S.W. 15

1922. Berkeley-Hill, Owen Alfred Rowland, M.A., M.D., B.Ch.Oxon, M.R.C.S., L.R.C.P.Lond., Lt.-Col. I.M.S., Medical Superintendent, Ranchi Mental Hospital for Europeans, Kanké, Bihar and Orissa, India.

1912. Berncastle, Herbert Melbourne, M.R.C.S., L.R.C.P.Lond., Medical Superintendent, Croydon Mental Hospital, Upper Warlingham, Surrey.

1927. Berry, Richard James Arthur, M.D.Edin.\&Melb., F.R.C.S., F.R.S.Edin., Director of Medical Services, Stoke Park Colony, Stapleton, Bristol ; "Rufford", Canford Lane, Westbury-on-Trym, Bristol.

1932. Bhathena, Sorab Jamasji, Lt.-Col. I.M.S., L.R.C.P.\&S.Edin., L.R.F.P.S.Glasg., L.M. Dubl., Civil Surgeon, Civil Hospital, Thana, Bombay, India. 
1920. Birch, William Somerset, M.C., M.R.C.S., L.R.C.P.Lond., Medical Superintendent, The Mental Hospital, Bridgetown; " Rosemont", Bridgetown, British West Indies.

I894. Blachford, James Vincent, C.B.E., M.D., B.S.Durh., M.R.C.S., L.R.C.P.Lond., M.P.C., Milverton House, Long Ashton, Somerset.

1898. Blair, David, M.A., M.D., C.M.Glasg., Medical Superintendent, Lancashire County Mental Hospital, Prestwich, Manchester. (Lect. on Ment. Dis., Univ. of Manchester.)

19r9. Blake, Stanley, L.R.C.P.\&S.Irel., Assistant Medical Officer, Grangegorman Mental Hospital, Dublin.

1932. Blyth, William, M.B., Ch.B.Glasg., Assistant Medical Officer, Riccartsbar Mental Hospital, Paisley.

r9oo. Bolton, Joseph Shaw, D.Sc., M.D., B.S., F.R.C.P.Lond., Medical Director, West Riding Mental Hospital, Wakefield. (Prof. of Ment. Dis., Univ. of Leeds.) (President, 1928-29.)

1922. Bostock, John, M.B., B.S., M.R.C.S., L.R.C.P.Lond., D.P.M., Lauriston, Wickham Terrace, Brisbane, Queensland, Australia.

r9r 8. Bower, Cedric William, L.M.S.S.A., Physician-Superintendent, Springfield House, near Bedford.

1926. Boyd, William, M.B., Ch.B.Edin., D.P.H., D.P.M., Medical Superintendent, Fife and Kinross District Asylum, Cupar, Fife.

1898. Boyle, A. Helen A., M.D.Brux., L.R.C.P.\&.S.Edin., 9, The Drive, Hove, Sussex.

1926. Braithwaite, Joseph, M.B., Ch.B.Edin., D.P.M., Assistant Medical Officer, Cumberland and Westmorland Mental Hospital, Garlands, Carlisle.

1922. Bramwell, Edwin, M.D., F.R.C.P.Edin. \& Lond., F.R.S.Edin., 23, Drumsheugh Gardens, Edinburgh. (Physician, Royal Infirmary, and Prof. of Clin. Med., Univ. of Edin.)

r9r 1. Brander, John, M.D., Ch.B.Edin., M.R.C.P.Lond., D.P.M., Medical Superintendent, Colney Hatch Mental Hospital, New Southgate, N. I I.

1925. Brennan, Richard Dominick, M.B., B.Ch.N.U.I., Assistant Medical Officer, District Mental Hospital, Waterford.

1930. Bristowe, Hubert C., M.D.Lond., M.R.C.S., L.R.C.P.L.ond., M.P.C., Hon. Major R.A.M.C. Medical Officer, Yatton Hall Home for Feeble-minded Children; Wrington, Somerset.

1922. Brock, Arthur John, M.D., Ch.B.Edin., Medical Superintendent, Garth Hill House, North Queensferry, Fife.

1924. Brown, Basil William, M.B., B.S.Lond., L.M.S.S.A., D.P.M., The Priory, Roehampton, London, S.W. I 5 .

1905. Brown, Harry Egerton, O.B.E., M.D., Ch.B.Glasg., M.P.C., 47, Yorkshire House, Field Street, Durban, Natal.

1908. Brown, Robert Dods, M.D., Ch.B., F.R.C.P.Edin., D.P.H., Dipl. Psych., PhysicianSuperintendent, Royal Mental Hospital, Aberdeen.

19r6. Brown, William, D.Sc.Lond., M.A., M.D., B.Ch.Oxon., F.R.C.P.Lond., 88, Harley Street, London, W. I. (Wilde Reader in Mental Philosophy, Univ. Oxford.)

1917. Bruce, Alexander Ninian, D.Sc., M.D., F.R.C.P.Edin., 8, Ainslie Place, Edinburgh. (Lect. on Neurol., Univ. of Edin.)

1932. Bruce, David Charles, M.B., Ch.B.Edin., Dipl. Psych. Edin., Medical Superintendent, Criminal Lunatic Department and State Institution for.Defectives, Perth.

1928. Bruce, David Kennedy, M.B., Ch.B.Glasg., D.P.M., Senior Assistant Medical Officer, Storthes Hall Mental Hospital, Kirkburton, near Huddersfield.

1893. Bruce, Lewis C., M.C., M.D., F.R.C.P.Edin., M.P.C., Medical Superintendent, Perth District Asylum, Druid Park, Murthly. (Secretary, Scottish Division, r9or-r9o7 Co-Editor of Journal, I9II-1916.)

1927. Brunlees, Oswald, L.R.C.P.\&S.Edin., 75, Denmark Villas, Hove, Sussex.

191 3. Brunton, George Llewellyn, M.D., Ch.B.Edin., Medical Superintendent, City Mental Hospital, Mapperley Hill, Nottingham.

1920. Bryce, William Henderson, M.B., C.M.Edin., Gifford Bank, Gifford, East Lothian.

1912. Buchanan, William Murdoch, M.B., Ch.B.Glasg., Medical Superintendent, Kirklands Mental Hospital, Bothwell, Lanarkshire. (Secretary, Scottish Division since 1920.)

1928. Burke, Noel Hawley Michael, M.R.C.S., L.R.C.P.Lond., D.P.M., D.M.R.E.Camb., Medical Superintendent, Cell Barnes Colony, St. Albans, Herts. 
1932. Burns, Robert Henderson, B.A.Cantab., M.R.C.S., L.R.C.P., Assistant Medical Officer, Claybury Mental Hospital, Woodford Bridge, Essex.

1924. Bushe, Charles Kendal, O.B.E., B.A., M.D., B.Ch.Dubl., Surg.-Capt. R.N., c/o Admiralty Medical Department, London, S.W. x.

1921. Buzzard, Sir Edward Farquhar, Bart., K.C.V.O., M.A., M.D., B.Ch.Oxon., F.R.C.P. Lond., Physician to H.M. The King; Regius Professor of Medicine, Oxford University ; 85, Banbury Road, Oxford.

1928. Calder, Flora Hannah Macdonald, M.A., M.D., D.P.M.Edin., I4, Dalhousie Street, Edinburgh.

192 I. Caldicott, Charles Holt, M.B.E., M.B., M.R.C.S., L.R.C.P.Lond., Grantbourne, Chobham, Surrey.

1928. Caldwell, William Alexander, M.R.C.S., L.R.C.P.Lond., D.P.M., Assistant Medical Officer, West Park Mental Hospital, Epsom.

1925. Cameron, Donald Ewan, M.B., Ch.B.Glasg., D.P.M., Brandon Hospital for Mental Diseases, Brandon, Manitoba, Canada.

1928. Cameron, Viola Cameron, M.R.C.S., L.R.C.P.Lond., D.P.M. Assistant Medical Officer, Springfield Mental Hospital Annexe, Kingston Road, New Malden, Surrey.

1897. Campbell, Robert Brown, M.D., C.M., F.R.C.P.Edin., Medical Superintendent, Stirling District Mental Hospital, Larbert. (Secretary, Scottish Division, 1910-20, and Chairman, 1929-30; President, 1932-33.)

1932. Cannon, Alexander, M.D., Ch.B., Ph.D., M.A., D.P.M., Assistant Medical Officer, Colney Hatch Mental Hospital, N. II.

1930. Cannon, H. C. A. Tandy, Surgeon Lt.-Commander R.N. (Ret.), L.R.C.P.\&S.I., Resident Physician, Moorcroft House, Hillingdon, Middlesex.

1927. Carey, Catherine, M.B., B.Ch.N.U.I., Assistant Medical Officer, Farnham House, Finglas, co. Dublin.

1905. Carre, Henry, L.R.C.P.\&S.Irel., Medical Superintendent, Glasgow District Mental Hospital, Woodilee, Lenzie, N.B.

1932. Carroll, Denis Charles, M.A.Camb., M.R.C.S., L.R.C.P., 22, Queen Anne Street, W. I.

1928. Cassin, Patrick Joseph, M.D., B.Ch.N.U.I., D.M.D., Assistant Medical Officer, Mental Hospital, Kilkenny, Ireland.

1922. Casson, Elizabeth, M.D., Ch.B.Brist., D.P.M., Dorset House, Clifton, Bristol.

1928. Cates, Henry Joseph, M.D.Lond., Medical Superintendent, Northwoods House, Winterbourne, Bristol.

1932. Chalmers, Edith, M.B., Ch.B.Glasg., Assistant Medical Officer, Chiswick House, Pinner, Middlesex.

I9r. Chambers, Walter Duncanon, M.A., M.D., Ch.B., F.R.C.P.Edin., M.P.C., Physician Superintendent, James Murray's Royal Asylum ; Murray House, Perth.

1928. Chapman, Theonie Renée, M.B., B.S.Durh., Assistant Medical Officer, Northumberland Mental Hospital, Morpeth.

1932. Chapman, William Noel James, M.B., Ch.B., D.P.H., Dipl. Psych.Edin., Assistant Medical Officer, Lanark District Asylum, Hartwood.

1928. Chennell, Eileen Anne, M.R.C.S., L.R.C.P.Lond., D.P.M., Assistant Medical Officer, Holloway Sanatorium, Virginia Water, Surrey.

1923. Chevens, Leslie Charles Frederick, M.R.C.S., L.R.C.P.Lond., D.P.M., Assistant Medical Officer, Cheshire County Mental Hospital, Parkside, Macclesfield.

1917. Chisholm, Percy, L.R.C.P.\&S.Edin., L.R.F.P.S.Glasg., Medical Superintendent, Queen Mary Hospital, Hammer Springs, New Zealand.

1907. Chislett, Charles Game Angus, M.B., Ch.B., F.R.F.P.S.Glasg., Medical Superintendent, Stoneyetts, Chryston, Lanark.

1921. Cholmeley, Montague Adye, M.R.C.S., L.R.C.P.Lond., D.P.M. (Retired), Heigham Hall, Norwich.

1920. Clark, Ronald Murray, M.B., C.M.Edin., 29, Irton Road, Southport.

1907. Clarke, Geoffrey, M.D.Lond., Medical Superintendent, London County Mental Hospital, Bexley, Kent.

1907. Clarkson, Robert Durward, B.Sc., M.D., C.M., F.R.C.P.Edin., Medical Officer, Royal Scottish National Institution; The Park, Larbert, Stirlingshire. (Lect. on Ment. Deficiency, Univ. of Edin.)

r931. Clegg, John Leonard, M.B., Ch.B., D.P.H., Assistant Medical Officer, South Yorkshire Mental Hospital, Sheffield. 
1925. Cobb, Geoffrey Francis, M.R.C.S., L.R.C.P.Lond., D.P.M., M.P.C., Deputy Medical Superintendent, The Manor, Epsom.

1900. Cole, Sydney John, M.A., M.D., B.Ch.Oxon., Medical Superintendent, Wilts County Mental Hospital, Devizes.

1930. Coleman, Stanley Maurice, M.R.C.S., L.R.C.P., D.P.M., Assistant Medical Officer, Dorset County Mental Hospital, Herrison, Dorchester.

1906. Collier, Walter Edgar, M.R.C.S., L.R.C.P.Lond., Senior Assistant Medical Officer, Kent County Mental Hospital, Barming Heath, Maidstone.

1903. Collins, Michael Abdy, O.B.E., M.D., B.S., M.R.C.S., L.R.C.P.Lond., Medical Superintendent, Kent County Mental Hospital, Chartham Downs. (General Secretary, 1912-18；Vice-Chairman, Educational Committee, 1919-27; Chairman, S.E. Division, since 1932.)

1910. Conlon, Thomas Peter, L.R.C.P.\&S.Irel., Resident Medical Superintendent, District Mental Hospital, Monaghan.

1921. Connell, Ernest Henry, M.B., Ch.B.Edin., D.P.M., 7, Greenhill Gardens, Edinburgh.

1920. Connell, Oliver George, M.C., L.R.C.P.\&S.Irel., Medical Superintendent, St. Andrew's Hospital, Thorpe, Norwich.

1914. Connolly, Victor Lindley, M.C., M.B., B.Ch.Belf., D.P.M., Medical Superintendent, Hants County Mental Hospital, Park Prewett, Basingstoke.

1927. Cook, Leslie Colin, M.R.C.S., L.R.C.P.Lond., D.P.M., Deputy Medical Superintendent, Fountain Hospital, Tooting Grove, S.W. 17.

19ro. Coombes, Percival Charles, M.R.C.S., L.R.C.P.Lond., Medical Superintendent, Surrey County Mental Hospital, Netherne, near Coulsdon.

1930. Cooper, Hugh Astley, M.B., B.S.Lond., M.R.C.S., L.R.C.P.Lond., D.P.M., Assistant Medical Officer, West Park Mental Hospital, Epsom.

1927. Cooper, James Woodman Astley, L.S.A., L.R.C.S., L.R.C.P., Medical Superintendent, Middleton Hall Private Mental Hospital, and Almora Hall, Middleton St. George, Durham.

1928. Copeland, Cecil Leonard, M.B., Ch.B.Liverp., D.P.M., Assistant Medical Officer, West Riding Mental Hospital, Wakefield.

1903. Cormac, Harry Dove, M.B., M.S.Madras, D.P.M., Medical Superintendent, Cheshire County Mental Hospital, Parkside, Macclesfield; Parkside House, Macclesfield. (Lect. on Ment. Dis., Univ. of Manch.) (Chairman, N. and M. Division, 1931-32.)

1891. Corner, Harry, M.D., M.R.C.S., L.R.C.P.Lond., M.P.C., Jersey Farm, Sandridge, near St. Albans, Herts.

I9 10. Coupland, William Henry, L.R.C.P.\&S.Edin., L.R.F.P.S.Glasg., Medical Superintendent, Royal Albert Institution; Albert House, Haverbreaks, Lancaster.

1932. Courtney, Patrick J., M.D.N.U.I., Assistant Medical Officer, Donegal Mental Hospital, Letterkenny.

1931. Couston, Alastair Gordon, L.R.C.P.\&S.Edin., Assistant Medical Officer, Cheshire County Mental Hospital, Chester.

1930. Cowie, Charles George, M.A., M.B., C.M., M.P.C., "Bonaccord", Westminster Road, Branksome Park, Bournemouth.

1911. Cox, Donald Maxwell, M.R.C.S., L.R.C.P.Lond., Deputy Medical Superintendent, Hereford County Mental Hospital, Burghill.

1926. Coyne, William Joseph, M.D., B.Ch.N.U.I., D.P.M., Physician, Chiswick House, Pinner ; "Clontarf", Barrow Point Avenue, Pinner, Middlesex.

1924. Craig, Alexander, M.B., Ch.B.Aberd., D.P.M., Assistant Physician, Royal Mental Hospital, Aberdeen.

1893. Craig, Sir Maurice, C.B.E., M.A., M.D., B.Ch.Camb., F.R.C.P.Lond., M.P.C. " Salona", East Preston, near Littlehampton, Sussex. (Secretary, Educational Committee, 1905-8; Chairman, Educational Committec, 191 2-19.)

1924. Craig, Roy Neville, M.D.Durh., M.R.C.S., L.R.C.P.Lond., D.P.M., 35, Southernhay West, Exeter.

1911. Crichlow, Charles Adolphus, M.B., Ch.B.Glasg., Senior Assistant Medical Officer, Bangour Village, Uphall, Linlithgowshire.

1930. Crichton-Miller, Hugh, M.A., M.D.Edin., M.D.Pavia, Hon. Director, The Institute of Medical Psychology ; 6, Devonshire Place, W. I.

1915. Crosthwaite, Frederick Douglas, M.B., Ch.B.Edin., D.P.H., Physician Superintendent, West Koppies Mental Hospital, Pretoria, South Africa.

1923. Crow, Norah Annie, M.A.Edin., M.D., B.S.Lond., "Kingsclere", Harrington Road, Brighton. 
1919. Cuthbert, James Harvey, M.B., Ch.B.Edin., D.P.M., Medical Superintendent, West Ham Mental Hospital, Goodmayes; "The Leazes", Barley Lane, Goodmayes, Essex.

r930. Cutts, George Lambert, M.R.C.S., L.R.C.P.Lond., L.D.S., D.P.M., Assistant Medical Officer, Long Grove Mental Hospital, Epsom, Surrey ; Farmfield, Horton Lane, Epsom.

1907. Daniel, Alfred Wilson, B.A., M.D., B.Ch.Camb., M.R.C.S., I..R.C.P.Lond., Medical Superintendent, Hanwell Mental Hospital, Southall, Middlesex. (Secretary, Educational Committce, 1920-27.)

1926. Darlington, Arthur, B.A., M.B., B.Ch.Dubl., D.P.H., D.P.M., Deputy Medical Superintendent, Somerset and Bath Mental Hospital, Wells.

1930. Das, Banarsi, M.B., B.S.Punjab, D.P.M., Civil Surgeon, Rae Bareli, U.P., India.

1896. Davidson, Andrew, M.D., C.M.Aberd., M.P.C., 221, Macquarie Street, Sydney, New South Wales.

1925. Davidson, Thomas Wishart, P.D., M.B., Ch.B.Glasg., D.P.M., Assistant Medical Officer and Pathologist, City Mental Hospital, Humberstone, Leicester.

1922. Davie, Thomas Macnaughton, M.C., M.D., Ch.B.Edin., Ph.D., D.P.M., Barrister-at-Law, Medical Superintendent, East Riding Mental Hospital, Beverley, Yorks.

1931. Davies, Sidney, M.B., B.S.Lond., Assistant Medical Officer, North Wales Counties Mental Hospital, Denbigh; Carn Ingli, Denbigh, N. Wales.

1928. Davies, Stuart Wyndham, M.R.C.S., L.R.C.P.Lond., D.P.M., Medical Officer, H.M. Prison Service; 162 , Ducane Road, London, W. 12.

I921. Davies-Jones, Charles William Saunderson, M.B., Ch.B.Edin., Medical Superintendent, Isle of Wight Mental Hospital, Whitecroft, Newport.

1920. Dawson, William Siegfried, M.A., M.D., B.Ch.Oxon., M.R.C.P.Lond., M.R.C.S.Eng., D.P.M., Prof. of Psychiat., University of Sydney, New South Wales, Australia.

1926. Deane, Frederick John, M.B., Ch.B.Edin., D.P.M., Medical Inspector, Ministry of Home Affairs, Northern Ireland; "Belmont ", Church Road, Belfast.

1925. Delany, John James, L..R.C.P.\&.S.Irel., 68, Wellington Road, Dublin.

1929. Denyssen, John Abrahain Felix, M.R.C.S., L.R.C.P.Lond,, Assistant Medical Officer, Banstead Mental Hospital, Sutton, Surrey.

1901. De Steiger, Adele Isabella, M.D.Lond., Dormansland, Lingfield, Surrey.

1905. Devine, Henry, O.B.E., M.D., B.S., F.R.C.P.Lond., M.R.C.S.Eng., M.P.C., Medical Superintendent, Holloway Sanatorium, Virginia Water, Surrey; The Ridge, Virginia Water, Surrey. [lect. on Psychol., Maudsley Hosp.] (Assistant Editor, 19161920, and Co-Editor of Journal, 1920-27.)

1904. Devon, James, I.R.C.P.\&S.Edin., F.R.F.P.S.Glasg., 67, Great King Street, Edinburgh.

1924. Devon, Martha Davidson, L.R.C.P.\&S.Edin., L.R.F.P.S.Glasg., Isle of Eigg, Invernessshire.

1932. Dewar, Douglas Campbell, L.R.C.P.\&S.Edin., L.R.F.P.S.Glasg., D.P.M.Lond., Assistant Medical Officer, Renfrew District Asylum, Dykebar, Paisley.

1925. Dhunjibhoy, Jal Edulji, M.B., B.S.Bomb., Major I.M.S., Medical Superintendent, The Indian Mental Hospital, Kanke, Ranchi, Bihar and Orissa, India ; c/o Lloyds Bank, 6, Pall Mall, S.W. I.

1921. Dick, Alexander, M.C., M.B., Ch.B.Glasg., Deputy Medical Superintendent, Glasgow District Mental Hospital, Woodilee, Lenzie, N.B.

1922. Dickson, James, M.C., M.B., Ch.B.Edin., "Whitehill”, The Broadway, Thorpe Bay.

I915. Dillon, Frederick, M.D., Ch.B.Edin., Medical Superintendent, Northumberland House, Finsbury Park, London, N. 4 .

1909. Dillon, Kathleen, L.R.C.P.\&S.Irel., Assistant Medical Officer, District Asylum, Mullingar.

1905. Dixon, J. Francis, M.A., M.D., B.Ch.Dubl., M.P.C., Medical Superintendent, City Mental Hospital, Humberstone, Leicester.

1879. Dodds, William John, D.Sc., M.D., C.M.Edin., 19, Marina Road, Prestwick, Ayrshire.

1892. Donelan, John O'Conor, L.R.C.P.\&.S.Irel., M.P.C., Medical Superintendent, Grangegorman Mental Hospital; St. Dymphna's, North Circular Road, Dublin. (Chairman, Irish Division, since 1931.) (Lect. on Ment. Dis., Univ. of Dubl.)

1928. Douglas-Morris, Ernest Mannering, L.M.S.S.A., Neurologist, Ministry of Pensions Clinic, Nottingham; Tykeford Abbey Nursing Home, Newport Pagnell, Bucks.

1919. Drake-Brockman, Henry George, M.R.C.S., L.R.C.P.Lond., Medical Superintendont, St. Luke's Hospital, Middlesbrough.

1916. Drummond, William Blackley, M.D., C.M., F.R.C.P.Edin., ro, Hartington Gardens Edinburgh. 
1921. Drury, Kenneth Kirkpatrick, M.C., B.A., M.D., B.Ch.Dubl., D.P.M., Medical Superintendent, Leicester and Rutland Mental Hospital, Narborough, near Leicester.

1907. Dryden, Arthur Mitchell, M.B., Ch.B.Edin., Medical Superintendent, Glasgow District Mental Hospital, Gartloch, Gartcosh.

1902. Dudgeon, Herbert Wm., M.D., B.S.Durh., M.R.C.S., L.R.C.P.Lond., Director of the Lunacy Department of Public Health, and Director of Abbassia Mental Hospital, Egypt.

1899. Dudley, Francis, L.R.C.P.\&S.Irel., c/o Barclay's Bank, Bodmin, Cornwall.

1926. Duncan, Archibald Glen, M.D., B.S., M.R.C.S., L.R.C.P.Lond., D.P.M., Assistant Medical Officer, Essex County Mental Hospital, Severalls, Colchester.

1923. Dunne, John, M.B., B.Ch.R.U.I., D.M.D., District Mental Hospital, Sligo.

1922. Dunscombe, Nicholas Dunscombe, M.A., M.B., B.Ch.Camb., L.M.S.S.A., D.P.H., Barrister at-Law, Medical Officer of Health; Town Hall, Bexhill, Sussex.

1932. Dymock, Thomas, M.B., Ch.B.Glasg., Assistant Medical Officer, Edinburgh District Mental Hospital, Bangour, West Lothian.

1906. Eager, Richard, O.B.E., M.D., Ch.B.Aberd., M.P.C., Medical Superintendent, Devon County Mental Hospital, Exminster.

1931. Earl, Charles James Cecil, M.R.C.P.I., D.P.M., Assistant Medical Officer, Caterham Mental Hospital, Caterham, Surrey.

I891. Earls, James Henry, M.D., M.Ch.R.U.I., L.S.A., D.P.H., M.P.C., Barrister-at-Law ; Fenstanton, Christchurch Road, Streatham Hill, London, S.W. 2.

1921. East, Guy Roland, M.D., B.S., B.Hy.Durh., D.P.H., Medical Superintendent, Northumberland County Mental Hospital, Cottingwood, Morpeth.

1907. East, Wm. Norwood, M.D., M.R.C.S., M.R.C.P.Lond., M.P.C., One of His Majesty's Commissioners of Prisons; Prison Commission, Home Office, Whitehall, Westminster, S.W. I.

1895. Easterbrook, Charles C., M.A., M.D., F.R.C.P.Edin., M.P.C., J.P., Physician-Superintendent, Crichton Roval Institution, Dumfries.

193I. Eaves, Elizabeth Cowper, M.D., B.S., D.P.M.Lond., Honorary Neuro-pathologist, South Yorkshire Mental Hospital, Wadsley, Sheffield. (Lect. on Physiol., Univ. of Sheffield.)

1924. Eddison, Herbert Wilfred, M.A., M.D.Camb., M.R.C.S., L.R.C.P.Lond., D.P.M., Medical Superintendent, Wonford House Hospital, Exeter.

1895. Edgerley, Samuel, M.A., M.D., C.M.Edin., M.P.C., Medical Superintendent, West Riding Mental Hospital, Menston, Leeds.

1897. Edwards, Francis Henry, M.D.Brux., M.R.C.P.Lond., "Cherchefelle ", Reigate, Surrey.

1924. Edwards, Thomas Lloyd, L.R.C.P.\&S.Edin., L.R.F.P.S.Glasg., D.P.M., Assistant Medical Officer, Glamorgan County Mental Hospital, Bridgend.

1919. Eggleston, Henry, M.B., B.S.Durh., M.P.C., I I Bungalow, The Orchards, Kemsing, Kent.

19or. Elgee, Samuel Charles, O.B.E., L.R.C.P.\&S.Irel., "Glendaragh", Sea Road, Cooden, Bexhill-on-Sea.

1889. Elkins, Frank Ashby, M.D., C.M.Edin., M.P.C., Hormead, 2 I, Parkside Drive, Watford, Herts.

1908. Ellison, Arthur, M.R.C.S., L.R.C.P.Lond., I 20, Domestic Street, Holbeck, Leeds.

1901. Erskine, Wm. J. Adams, M.D., C.M.Edin., The Orchard, Farnby, near Liverpool.

1925. Esson, Walter Louis, M.A., M.B., Ch.B.Aberd., 21 , Oldham Road, Miles Platting, Manchester.

1895. Eurich, Frederick Wilhelm, M.D., C.M.Edin., Lanshawe Cottage, Ilkley, Yorks. (Prof. of For. Med., Univ. of Leeds.)

1909. Eustace, William Neilson, L.R.C.P.\&S.Irel., Resident Medical Officer, "Lisrouagh", Glasnevin, Dublin.

1918. Evans, Albert Edward, M.B., B.S.Lond., M.R.C.S., L.R.C.P.Lond., D.P.H., Commissioner, Board of Control, II, Weymouth Avenue, Mill Hill, N.W. 7.

1927. Ewan, Grey Lamont, J.P., B.Sc., M.B., Ch.M.Sydney, D.P.M., Medical Superintendent, The Mental Hospital, Stockton, nr. Newcastle, New South Wales.

i 891. Ewan, John Alfred, M.A.St.And., M.D., C.M.Edin., M.P.C., Greylees, Godalming, Surrey.

1930. Ewen, John Harold, M.R.C.S.Eng., L.R.C.P.L.ond., D.P.M., Assistant Medical Officer, Surrey County Mental Hospital, Netherne, Coulsdon.

1914. Ewing, Cecil Wilmot, L.R.C.P.\&S.Irel., D.P.M., Medical Superintendent, Storthes Hall Mental Hospital, Kirkburton, nr. Huddersfield. 
1929. Fairbairn, William Ronald Dodds, M.A., M.D., Ch.B.Edin., Dipl. Psych., Psychologist, Jordanburn Nerve Hospital; I8, Lansdowne Crescent, Edinburgh. (Lect. on Psych., Univ. of Edin.)

1925. Fairweather, Anne, M.D., B.S.Durh., D.P.M., The Red House, Haydon Bridge, Northumberland.

1908. Fenton, Henry Felix, M.B., Ch.B.Edin., Medical Superintendent, Worcester County and City Mental Hospital, Powick.

1930. Fenwick, Philip Cuthbert Collingwood, L.M.S.S.A., Assistant Medical Officer, East Sussex Mental Hospital, Hellingly.

1928. Finiefs, Leonidas Aristodimos, M.R.C.S., L.R.C.P.Lond., M.D.Paris, Assistant Medical Officer, Three Counties Mental Hospital, Arlesey, Beds.

1889. Finlay, David, M.D., C.M.Glasg., Medical Superintendent, Glamorgan Mental Hospital, Bridgend.

1906. Firth, Arthur Harcus, M.A., M.D., B.Ch.Edin., Deputy Medical Superintendent, Worcester County Mental Hospital, Barnsley Hall, Bromsgrove.

19 30. Fisher, John William, M.R.C.S., L.R.C.P., D.P.H., D.P.M., Temporary Assistant Medical Officer, Devon Mental Hospital; Sunny Cottage, Shaldon, near Teignmouth, S. Devon.

1903. Fitzgerald, Alexis, L.R.C.P.\&S.Irel., Medical Superintendent, District Mental Hospital, Waterford.

1929. FitzGerald, Edward Joseph, M.B., B.Ch.N.U.I., Assistant Medical Officer, Lancashire County Mental Hospital, Winwick, Warrington.

1908. Fitzgerald, James Francis, L.R.C.P.\&.S.Irel., Assistant Medical Officer, District Mental Hospital, Clonmel.

1928. Fitzgerald, John, M.B., B.Ch.N.U.I., M.D., D.M.D., Assistant Medical Officer, Grangegorman Mental Hospital, Dublin.

I930. Fitzgerald, John Joseph, M.D.Durh.\&Brux., Assistant Physician, Mental Hospital, Cork; Mile House, Cork.

1930. Fitzgerald, Nora May, M.B., B.Ch., B.A.O., Grangegorman Mental Hospital, Dublin.

1921. Fleming, Gerald William Thomas Hunter, M.R.C.S., L.R.C.P.Lond., D.P.M., Medical Superintendent, Hereford County and City Mental Hospital, Burghill, Hereford. (.Assistant Editor, 1931-32, and Co-Editor of Journal since 1932.)

1904. Fleming, Wilfrid Louis Remi, M.R.C.S., L.R.C.P.Lond., J.P., Suffolk House, Pirbright, Surrev.

I932. Flemyng, George Gilbert, M.D.Dubl., (retired), 37c, Maresfield Gardens, London, N.W. 3.

1925. Flind, James, M.B., Ch.B.Glasg., Senior Assistant Medical Officer, Peckham House, London, S.E. I5.

1930. Forbes, Donald John, M.B., Ch.B.St.And., Medical Superintendent, Baldovan Certified Institution, Craigmill House, by Dundee.

1927. Ford-Robertson, William Marsden, M.D., Ch.B.Edin., Director, West of Scotland NeuroPsychiatric Research Institute; 28, Hamilton Park Avenue, Glasgow, W. 2.

I9r I. Forrester, Archibald Thomas William, M.D., B.S., M.R.C.S., L.R.C.P.Lond., Medical Superintendent, Warwick County Mental Hospital, Hatton.

1926. Forrester, Robert Cairns, M.B., Ch.B.Edin., Assistant Medical Officer, City of London Mental Hospital, Stone, Dartford.

1916. Forsyth, Charles Wesley, M.D., M.R.C.S., L.R.C.P.Lond., Medical Superintendent, Winson Green Mental Hospital, Birmingham. (Clin. Lect. on Ment. Dis., Univ. of. Birm.)

191 3. Forward, Ernest Lionel, M.R.C.S., L.R.C.P.Lond., D.C.M.S. Ministry of Pensions, I, Sanctuary Buildings, London, S.W. I.

1930. Fox, Edward Arthur Haslam, M.B., Ch.B., D.P.M., Assistant Medical Officer, City Mental Hospital, Fulford, York.

1925. Fox, Francis Elliot, B.A.Camb., M.R.C.S., L.R.C.P.I.ond., Medical Officer, Brislington House, Bristol.

1920. Fox, J. Tylor, M.A., M.D., B.Ch.Camb., M.R.C.S., L.R.C.P.Lond., D.P.M., Medical Superintendent, Lingfield Epileptic Colony; The Homestead, Lingfield, Surrey.

1923. Franklin, Marjorie Ellen, M.B., B.S., M.R.C.S., L.R.C.P.Lond., D.P.M., Assistant, London Clinic for Psycho-Analysis; 3, Bulstrode Street, Welbeck Street, London, W. I.

1919. Fraser, Kate, B.Sc., M.D., Ch.B.Glasg., D.P.H., Deputy Commissioner, General Board of Control, Scotland; 25, Palrrerston Place, Edinburgh. 
1928. Fraser, William, B.Sc., M.B., Ch.B.Glasg., D.P.H., D.P.M., Deputy Medical Superintendent, North Riding Mental Hospital, York.

1921. Fuller, Hugh Hercus Cavendish, M.B., Ch.B.Edin., I 36, Inverness Terrace, W. 2.

1902. Fuller, Lawrence Otway, M.R.C.S., L.R.C.P.Lond., Medical Superintendent, Three Counties Mental Hospital, Arlesey, Beds.

1928. Gall, Herbert, M.R.C.S., L.R.C.P.Lond., Major R..A.M.C., British Military Hospital, Quetta, India.

1928. Gallagher, Charles Eddie, M.R.C.S., L.R.C.P.Lond., Talfourd House, 28, Mount Pleasant Road, Hastings.

1932. Galloway, James Forbes, M.D.Liverp., D.P.H., Health Department, Town Hall, Chester.

1906. Gane, Edward Palmer Steward, M.D.Durh., M.R.C.S., L.R.C.P.Lond., Castle Green, Llansawel, Llandilo, Carmarthen.

1928. Gardner, Dorothy Margaret, M.B., B.Ch.Belf., D.P.H., Assistant Medical Officer, Belfast Mental Hospital, Purdysburn, Belfast.

1931. Garrod, Marjorie, M.R.C.S., L.R.C.P., Hon. Medical Registrar, The Institute of Medical Psychology ; "Bankcroft", Douglas Road, Harpenden, Herts.

1912. Garry, John William, M.B., B.Ch.N.U.I., Assistant Medical Superintendent, District Mental Hospital, Ennis, co. Clare.

1912. Gavin, Lawrence, M.B., Ch.B., L.R.C.P.\&.S.Edin., L.R.F.P.S.Glasg., Medical Superintendent, District Asylum, Mullingar.

1896. Geddes, John William, M.B., C.M.Edin., 7, Bedford Row, Worthing.

1930. Gemmell, Daniel Topping, L.R.C.P.\&S.I., L.R.T.P.\&S.G., D.P.M., Dornhurst, Halstead, Essex.

1919. Gifford, John, B.A.Cape, M.B., Ch.B.Edin., D.P.M., Deputy Medical Superintendent, Lancashire County Mental Hospital, Winwick, Warrington.

1931. Gilchrist, Thomas, L.R.C.P.\&.S.Edin., L.R.C.P.S.Glasg., Assistant Medical Officer, Park Prewett Mental Hospital, Basingstoke.

1899. Gilfillan, Samuel James, O.B.E., M.A., M.B., C.M.Edin., Medical Superintendent, Bailbrook House, Bath.

1931. Gill, Samuel Ernest, M.D.Lond., D.P.M., Commissioner, Board of Control ; 89, Coombe Road, Croydon.

1923. Gillespie, Isabella Annie, M.B., B.Ch.Edin., D.P.M., Senior Assistant Medical Ofticer, Cheshire County Mental Hospital, Upton, Chester.

192 I. Gillespie, Robert Dick, M.D., Ch.B.Glasg., M.R.C.P.Lond., D.P.M., 49, Wimpole Street, W. I. (Lect. on Psychiat. Med., Guy's Hosp.)

1920. Gillis, Kurt, M.B., Ch.B.Edin., Assistant Physician-Superintendent, Mental Hospital, Bloemfontein, South Africa.

1897. Gilmour, John Rutherford, M.B., C.M., F.R.C.P.Edin., M.P.C., Medical Superintendent, West Riding Mental Hospital, Scalebor Park, Burley-in-Wharfedale, Yorks. (Secretary, N. and M. Division, 1920-28, and Chairman, 1929-31.)

I906. Gilmour, Richard Withers, M.B., B.S.Durh., M.R.C.S., L.R.C.P.Lond., Phrsician-inCharge, Woodside Nerve Hospital, Muswell Hill ; 39, Harley Street, London, W. I. (Lect. on Psychiat., Middx. Hosp.)

1929. Glaister, John Norman, M.B., B.S., M.R.C.S., L.R.C.P.Lond., Assistant Medical Officer, Northumberland House; Physician, British Hospital for Nervous and Mental Disorders ; 2, Devonshire Place, London, W. I.

1927. Goitein, Percy L., M.B., B.S., M.R.C.S., L.R.C.P.Lond., D.P.M., Deputy Medical Superintendent, City Mental Hospital, Canterbury.

1923. Golla, Frederick Lucien, M.A., M.B., B.Ch.Oxon., F.R.C.P.Lond., Director of the Laboratory and Pathologist, London County Mental Hospitals, The Maudsley Hospital, Denmark Hill, London, S.E. 5 ; The Dene, Sunninghill, Berks. (Chairman, Research and Clinical Committee since 1931.)

1897. Good, Thomas, Saxty O.B.E., M.A.Oxon., M.R.C.S., L.R.C.P.Lond., Medical Superintendent, County and City Mental Hospital, Littlemore, Oxford. (Lect. on Psychiat., Univ. of Oxford.) (PResident, 1930-31.)

1889. Goodall, Edwin, C.B.E., M.D., B.S., F.R.C.P.Lond., M.P.C., " Fairlawn", Kingsway, Hove, Sussex. (Assistant Editor, 1894-5, and Co-Editor of Journal, 1895-98; President, 1923-24.)

1920. Gordon, George, M.B., B.Ch.Glasg., 56, Anne Boleyn's Walk, Cheam, Surrey.

1928. Gordon, Ronald Grey, D.Sc., M.D., F.R.C.P.Edin., 9, The Circus, Bath.

rgor. Gostwyck, Cecil Hubert Gostwyck, M.B., Ch.B., F.R.C.P.Edin., Dipl. Psych., M.P.C., Deputy Medical Superintendent, Rampton State Institution, Retford, Notts. 
1923. Gough, Isabel Falconer, M.B., Ch.B., L.R.C.P.\&S.Edin., L.R.F.P.S.Glasg., D.P.M., Three Corner Park, Calstock, Cornwall.

1928. Grace, Pierce, L.R.C.P.\&S.I., Resident Medical Superintendent, District Mental Hospital, Portlaoighise (Maryborough), Leix.

1914. Graham, Norman Bell, M.C., B.A.R.U.I., M.B., B.Ch.Belf., D.P.M., Senior Assistant Medical Officer, Purdysburn Villa Colony, Belfast.

19r8. Graham, Samuel John, L.R.C.P.\&S.Edin., L.R.F.P.S.Glasg., Resident Medical Superintendent, Purdysburn Villa Colony, Belfast.

r9o8. Graham, William Shepherd, M.B., B.Ch.R.U.I., Senior Assistant Medical Officer, Somerset and Bath Mental Hospital, Cotford, near Taunton.

1921. Grant, Alastair Robertson, M.D., Ch.B.Aberd., Medical Superintendent, Lancashire County Mental Hospital, Whittingham, Preston.

1927. Grant, Florence Margaret (née Gamble), 306, St. James's Court, Buckingham Gate, S.W. I.

1925. Grant, John King, M.B., Ch.B.Aberd., Assistant Physician, Royal Asylum, Montrose.

1930. Grant, Penuel Grant, M.B., Ch.B., D.P.H., Assistant Medical Officer, City Mental Hospital, Fishponds, Bristol.

r9r5. Graves, Thomas Chivers, B.Sc., M.D., B.S.Lond., F.R.C.S.Eng., L.R.C.P.Lond., M.R.C.V.S., Medical Superintendent, Rubery Hill and Hollymoor Mental Hospitals, Northfield, Birmingham.

1916. Gray, Cyril, L.R.C.P.\&S.Edin., L.R.F.P.S.Glasg., Senior Assistant Medical Officer, City Mental Hospital, Gosforth, Newcastle-upon-Tyne.

1928. Gray, James, M.B., Ch.B.Edin., " Jemimaville”, Poyntzfield-by-Cononbridge, Ross-shire.

I921. Gray, Joseph Anthony Wenceslaus Pereira, M.D.Brux., M.R.C.S., L.R.C.P.Lond., Visitor of Licensed Houses ; 3, Northernhay Place, Exeter.

1909. Greene, Thomas Adrian, L.R.C.P.\&S.Irel., J.P., Medical Superintendent, District Mental Hospital, Carlow.

1922. Gregorson, Albert William, M.D., Ch.B., F.R.F.P.S.Glasg., Physician and Deputy Superintendent, North Middlesex Hospital, Edmonton, London, N. I8; I1 2, Brownlow Road, New Southgate, N. II.

1927. Grierson, Hugh Arrowsmith, M.C., M.B., B.S.Lond., Medical Officer, H.M. Prison, Brixton, S.W. 2.

1926. Griffiths, Gwenvron Mary, M.D., M.R.C.P.Lond., 50, Wimpole Street, London, W. I.

19or. Grills, Galbraith Hamilton, M.D., B.Ch.R.U.I., D.M.D., M.P.C., Medical Superintendent, Cheshire County Mental Hospital, Upton, Chester.

1923. Grossman, Simon, M.R.C.S., L.R.C.P.Lond., c/o South Africa House, Strand, W.C. 2.

1922. Guppy, Francis Henry, M.C., M.R.C.S., L.R.C.P.Lond., D.P.M., Deputy Medical Superintendent, Brighton Mental Hospital, Haywards Heath, Sussex.

1932. Hagedorn, Egmont Oscar, L.R.C.P.\&.S.Edin., L.R.F.P.S.Glasg., Assistant Medical Officer, Stirling District Mental Hospital, Larbert.

1894. Halsted, Harold Cecil, M.D.Durh., L.S.A., M.R.C.S., L.R.C.P.Lond., Manor Road, Selsey, Sussex.

1928. Hamilton, John Gerard, M.B., B.S., M.R.C.S., L.R.C.P.Lond., D.P.M., Assistant Medical Officer, Holloway Sanatorium, Virginia Water, Surrey.

1920. Hancock, Allen Coulter, M.C., M.B., B.S., M.R.C.S., L.R.C.P.Lond., D.P.H., D.P.M., Medical Superintendent, Kent County Mental Hospital, Barming Heath, Maidstone.

1929. Handley, Richard, M.B., Ch.B.Manch., D.P.M., Medical Director, David Lewis Epileptic Colony, Warford, Alderley Edge, Cheshire.

1930. Hannesson, Hannes, B.Sc., M.R.C.S., L.R.C.P.Lond., Authors' Club, Whitehall Court, S.W. I.

1923. Hardcastle, Douglas Noël, M.R.C.S., L.R.C.P.Lond., D.P.M., Mountgrove, ro2, Fitzjohn's Avenue, Hampstead, London, N.W.

1920. Harding, Edward Palmer, L.R.C.P.\&S.Irel., Deputy Medical Superintendent, East Riding Mental Hospital, Beverley.

1932. Harkins, William, M.A., M.B., Ch.B.Aberd., Assistant Medical Officer, West Riding Mental Hospital, Storthes Hall, Kirkburton, near Huddersfield.

1920. Harper, Raymond Sydnev, M.R.C.S., L.R.C.P.Lond.; 36, First Avenue, Hove, Sussex.

r904. Harper-Smith, George Hastie, M.A., M.D.Camb., M.R.C.S., L.R.C.P.Lond., Medical Superintendent, Brighton Mental Hospital; Galagate, Haywards Heath, Sussex.

1931. Harris, John Borrie, L.R.C.P.\&S.Edin., L.R.F.P.S.Glasg., Assistant Physician, Kingseat Mental Hospital, Newmachar, Aberdeenshire. 
1924. Harris, John Stuart, M.D., Ch.B.Edin., M.R.C.P.L.ond., D.P.M., Deputy Medical Superintendent, West Park Mental Hospital, Epsom.

1928. Harris, Noel Gordon, M.B., B.S., M.R.C.S., L.R.C.P.Lond., D.P.M., Assistant Medical Officer, Springfield Mental Hospital, near Tooting, S.W. I7.

1898. Harris-Liston, Llewellyn, M.D.Brux., L.S.A., M.R.C.S., L.R.C.P.Lond., Fairholm, Cobden Street, Darlington.

1930. Harrison, Geoffrey J., L.R.C.P.\&S.I., Assistant Medical Officer, St. Edmondsbury, Lucan, co. Dublin.

1928. Harrowes, William McConnachie, M.D., Ch.B.Glasg., D.P.M., Medical Superintendent, New Saughton Hall, Polton, Midlothian.

1905. Hart, Bernard, M.D., F.R.C.P.Lond., M.R.C.S.Eng., I, Harcourt House, Cavendish Square, London, W. I. (Lect. on Ment. Dis., Univ. Coll. Hosp.)

1892. Haslett, William John Handfield, M.R.C.S., L.R.C.P.Lond., M.P.C., J.P., Resident Medical Superintendent, Halliford House, Sunbury-on-Thames.

1930. Haworth, Norah Alice, M.A.Camb., M.R.C.S.Eng., L.R.C.P.Lond., D.P.M., Assistant Medical Officer, Severalls Mental Hospital, Colchester.

1923. Hayes, Edmund Duncan Tranchell, B.A., M.D., B.Ch.Dubl., D.P.M., Senior Assistant Medical Officer, County Mental Hospital, Berry Wood, Northampton.

1924. Hayes, Henry Douglas, M.D., Ch.B.Edin., D.P.M., The Mental Hospital, Porirua, Wellington, New Zealand.

1920. Haynes, Horace Guy Lankester, M.R.C.S., L.R.C.P.Iond., Littleton Hall, Brentwood, Essex. (Sccretary, Parliamentary Committee since I929.)

1927. Healey, Frederick Henry, B.Sc., M.B., Ch.B.Birm., D.P.M., Deputy Medical Superintendent, Borough Mental Hospital, Rowditch, Derby.

1932. Hemphill, Robert Edward, M.B., B.Ch., B.A.O.Dubl., Assistant Medical Officer, City Mental Hospital, Bristol.

1920. Henderson, Cyril John, M.B.Durh., Assistant Medical Officer, The Royal Albert Institution, Lancaster.

1929. Henderson, David, M.D., Ch.B.Glasg., Assistant Medical Officer and Pathologist, Stafford County Mental Hospital, Cheddleton; The Hollies, Cheddleton, near Leek.

1916. Henderson, David Kennedy, M.D., Ch.B.Edin., F.R.F.P.S.Glasg., Physician Superintendent, Edinburgh Royal Mental Hospital, Morningside ; Tipperlinn House, Edinburgh. (Professor of Psychiatry, University of Edinburgh.)

1905. Henderson, George, M.A., M.B., Ch.B.Edin., 25, Commercial Road, Peckham, London, S.E. 15 .

1923. Henderson, Norman Keane, B.A., LL.B.Camb., M.B., Ch.B.Edin., D.P.H., D.P.M. Medical Superintendent, Kesteven Mental Hospital, Greylees, Sleaford, Lincs.

1930. Hennelly, Thomas John, M.D., B.Ch., N.U.I., D.P.M., Assistant Medical Officer, Surrey County Mental Hospital, Brookwood, Woking.

1924. Hensman, Henry Saumarez, L.M.\&S.Madras, M.R.C.S., L.R.C.P.Lond., M.P.C., Medical Superintendent, Government Mental Hospital, Kilpauk, Madras.

1924. Herbert, James Edward, M.B., B.Ch.R.U.I., Senior Assistant Medical Officer, District Asylum, Omagh.

1925. Heron, John, M.B., Ch.B.Edin., D.P.M., 78, Bonaly Road, Edinburgh.

1929. Hewitt, Edward John Campbell, M.B., Ch.B.Edin., D.P.M., Assistant Medical Officer and Pathologist, Napsbury Mental Hospital, St. Albans, Herts.

1912. Higson, William Davies, M.B., Ch.B.Liverp., D.P.H., Medical Officer, H.M. Prison, Liverpool.

1931. Hills, Harold William, M.D., B.S.Lond., M.R.C.P., D.P.M.Camb., Honorary Physician and Anæsthetist, Stroud General Hospital ; "Cotsmoor", Rodborough Common, Stroud, Glos.

1932. Hingston, Cicely Lamorna, M.R.C.S., L.R.C.P., M.B.E.(Mil.), Hon. Visiting Physician, Lady Chichester Hospital, Hove ; 9, The Drive, Hove, Sussex.

1900. Hollander, Bernard, M.D.Freib., M.R.C.S., L.R.C.P.Lond., 57, Wimpole Street, London, W. I.

1929. Holmes, Eric Gordon, M.A., M.B., B.Ch.Camb., Assistant to the Downing Professor of Medicine, Demonstrator of Pharmacology, Cambridge ; Pharmacological Laboratory, Cambridge.

1925. Honan, Bernard Francis, L.R.C.P.\&S.Irel., D.P.M., Medical Superintendent, District Mental Hospital, Cork.

1920. Hooper, Reginald Arthur, M.B., B.S.Durh., Medical Superintendent, City Mental Hospital, Fulford, York. 
1929. Hopkins, Edwin Lancelot, M.R.C.S., L.R.C.P.Lond., D.P.H., D.P.M., Medical Superintendent, County Mental Hospital, Mickleover, Derby.

1926. Hopwood, Joseph Stanley, M.B., B.S., M.R.C.S., L.R.C.P.Lond., Medical Officer, State Criminal Lunatic Asylum, Broadmoor.

1926. Horton, Phyllis Mary, M.R.C.S., L.R.C.P.Lond., D.P.M., Wye House, Buxton.

I918. Horton, Wilfred Winnall, M.D., C.M.Edin., Medical Superintendent, Wye House, Buxton.

1926. Hosie, William, M.B., Ch.B.Glasg., 19, Lanfine Road, Paisley, Renfrewshire.

1 894. Hotchkis, Robert Dunmore, M.A.Glasg., M.D., B.S.Durh., M.R.C.S., L.R.C.P.Lond., M.P.C., Medical Superintendent, Renfrew District Mental Hospital, Dykebar, Paisley.

1900. Hughes, Percy T., M.B., C.M.Edin., D.P.H., Medical Superintendent, Worcester County Mental Hospital, Barnesley Hall, Bromsgrove. (Lect. on Ment. Dis., Univ. of Birm.)

1904. Hughes, William Stanley, M.B., B.S., M.R.C.S., L.R.C.P.Lond., Medical Superintendent, Salop County Mental Hospital, Bicton Heath, Shrewsbury.

1932. Hull, Eric Randal, M.B., B.Ch., B.A.O., Assistant Medical Officer, County Mental Hospital, Lancaster.

193I. Hunter, Constance Primrose Helena, L.R.C.P.\&S.Edin., D.P.H.Edin., Secretary, Scottish Association for Mental Welfare, 25, Palmerston Place, Edinburgh; St. Catherine's, Linlithgow.

1897. Hunter, David, M.A., M.B., B.Ch.Camb., L.S.A., Medical Superintendent, The Coppice, Nottingham. (Secretary, S.E. Division, 1910-1913).

1928. Hunter, James Dewar Hunter, M.B., Ch.B.N.Z., Dipl. Psych., Senior Assistant Medical Officer, Mental Hospital, Seacliffe, Otago, New Zealand.

1904. Hunter, Percy Douglas, M.R.C.S., L.R.C.P.Lond., D.P.M., Deputy Medical Superintendent, Three Counties Mental Hospital, Arlesey, Beds.

1931. Hutton, Eustace, M.R.C.S., L.R.C.P., Deputy Medical Superintendent, North Wales Counties' Mental Hospital, Denbigh.

I9I1. Hutton, Isabel Emslie, M.D., Ch.B.Edin., Hon. Physician, British Hospital for Mental and Nervous Disorders; 6, Montagu Place, London, W. I.

1888. Hyslop, Theo. Bulkeley, M.D., C.M., M.R.C.P., L.R.C.S., F.R.S.Edin., M.P.C., Stretton House, Church Stretton, Salop. (Chairman, Library Committee, 1926-27.)

1926. Illingworth, Reginald Ernest, L.R.C.P.\&S., L.D.S.Edin., D.Psy.Durh., Deputy Medical Superintendent, Northumberland County Mental Hospital, Cottingwood, Morpeth.

1915. Ingall, Frank Ernest, L.R.C.P.Lond., F.R.C.S.Eng., D.P.H., Public Health Offices, Clarence Street, Southend-on-Sea.

1926. Ironside, Archibald Jennings, M.A., M.B., Ch.B.Aberd., Queenstown Mental Hospital, Cape Province, South Africa.

1932. Irvine, Francis Philip, M.B., Ch.B.Liverp., Assistant Medical Officer, East Sussex Mental Hospital, Hellingly.

I906. Irwin, Peter Joseph, L.R.C.P.\&S.Irel., Medical Superintendent, District Mental Hospital, Limerick.

1920. Jackson, John Luke, M.B., B.Ch.Belf., Medical Superintendent, Hants County Mental Hospital, Knowle, Fareham.

1927. Jacob, Frank Harwood, M.D., F.R.C.P.Lond., Hon. Physician, General Hospital; 32, Regent Street, Nottingham.

1927. Jacobson, Jack Nathan, M.R.C.S., L.R.C.P.Lond., D.P.M., Assistant Medical Officer, Tooting Bec Mental Hospital, London, S.W. I7.

1914. James, George William Blomfield, M.C., M.D., B.S.Lond., L.S.A., D.P.M., Physician, Moorcroft House, Hillingdon, Uxbridge; 124 , Harley Street, W. I. (Lect. on Ment. Dis., St. Mary's Hosp.) (Secretary, Parliamentary Committee, 1926-29).

1931. Jamieson-Craig, Catherine Isabella Anderson, M.A., M.B., Ch.B.Aberd., Assistant Medical Officer, Lancashire County Mental Hospital, Whittingham, Preston.

1922. Jarrett, Reginald Fitzroy, F.R.F.P.S.Glasg., L.M.S.S.A., Medical Superintendent, Leybourne Grange Colony, West Malling, Kent.

1908. Jeffrey, Geo. Rutherford, M.D., Ch.B.Glasg., F.R.C.P., F.R.S.Edin., M.P.C., Medical Superintendent, Bootham Park, York.

1925. Jenkins, John Alexander, M.B., Ch.B.Glasg., Senior Assistant Medical Officer, Mental Hospital, Larbert. 
1924. Jenkins, Reginald Edward, L.M.S.S.A., Egmont House, Brighton Road, Sutton.

1893. Johnston, Gerald Herbert, L.R.C.P.\&S.Edin., L.R.F.P.S.Glasg., Brooke House, Upper Clapton, London, E. 5 .

1928. Johnston, James McLaughlan, M.B., Ch.B.Edin., Medical Officer, H.M. Prison Service 156, Ducane Road, London, W. I 2.

1905. Johnston, Thomas Leonard, L.R.C.P.\&S.Edin., L.R.F.P.S.Glasg., The Gabled House, Leadenham, Lincs.

1927. Kearney, Joseph, M.B., B.Ch.N.U.I., Portrane Mental Hospital, Donabate, Co. Dublin.

1886. Keay, John, C.B.E., M.D., C.M.Glasg., F.R.C.P.Edin., "Champions", Beaminster, Dorset. (PRESIDENT, I9I8; Chairman, Educational Committce, I920-27.)

1907. Keene, George Henry, M.D., B.Ch.Dubl., Medical Superintendent, Stewart Institution, Palmerston; I 4, Palmerston Park, Dublin.

r9o9. Keith, William Brooks, M.C., M.D., Ch.B.Aberd., M.P.C., Medical Superintendent, St. Audry's Hospital, Melton; Redwald House, Melton, Suffolk. (Secretary, Parliamentary Committee, 1921-26.)

1924. Kelly, Daniel Lane, L.R.C.P.\&S.Irel., Inspector of Mental Hospitals, Local Government Department ; 52, Grosvenor Road, Rathgar, Dublin.

1930. Kelly, John Vincent, M.B., B.Ch., D.P.M., Assistant Medical Officer, District Mental Hospital, Castlebar, Ireland.

1931. Kennedy, Robert Stewart, M.B., Ch.B., Assistant Medical Officer, Cheshire County Mental Hospital, Chester.

1897. Kerr, Hugh, M.A., M.D., C.M.Glasg., Medical Superintendent, Bucks County Mental Hospital, Stone, Avlesbury.

1902. Kerr, Neil Thomson, M.B., C.M.Edin., J.P., Medical Superintendent, Lanark District Mental Hospital, Hartwood, N.B. (Chairman, Scot. Div., r930-3I.)

1920. Key, Gordon James, M.B., Ch.B.Aberd., Medical Superintendent, Mental Hospital, Grahamstown, C.P., South Africa.

1923. el Kholy, Mohamed Kamel, M.R.C.S., L.R.C.P.Lond., D.P.M., Medico-Legal Department, Bab-el-Khalk, Cairo, Egypt.

1920. Kimber, William Joseph Teil, M.R.C.S., L.R.C.P.Lond., D.P.M., Medical Superintendent, Herts County Mental Hospital, Hill End, St. Albans. (Secretary, Educational Committee, 1927-30.)

1903. King, Frank Raymond, B.A.Camb., M.R.C.S., L.R.C.P.Lond., Medical Superintendent, Peckham House, Peckham, London, S.E. I 5.

1902. King-Turner, Arthur Charles, M.B., C.M.Edin., Medical Superintendent, The Retreat, Fairford, Gloucestershire.

1915. Kirwan, Richard R., M.B., B.Ch.R.U.I., Assistant Medical Officer, West Riding Mental Hospital, Menston, Leeds.

1919. Knight, Mary Reid, M.A., M.B., Ch.B.Glasg., Assistant Medical Officer, Paisley Mental Hospital, Riccartsbar.

1932. Krausz, Desider, M.D.Hungary, M.D.Bologna, Assistant Medical Officer, East Sussex Mental Hospital, Hellingly:

1914. Ladell, Robert George Macdonald, M.B., Ch.B.Vict., 395, Coventry Road, Small Heath, Birmingham.

1923. Laing, John Kidd Collier, M.B., B.S.Melb., D.P.M., Medical Superintendent, Darenth Training Colony, Dartford, Kent.

1925. Landers, John Joseph, M.B., B.Ch.N.U.I., D.P.H., Medical Ofticer, H.M.Prison ; 164, Ducane Road, London, W. 12.

1896. Langdon-Down, Reginald L., M.A., M.B., B.Ch.Camb., Normansfield, Hampton Wick, Middlesex.

19r9. Langton, Peregrine Stephen Brackenbury, M.B., B.S., M.R.C.S., L.R.C.P.Lond., Medical Superintendent, Roval Earlswood Institution, Redhill, Surrey.

1925. Lascelles, William James, M.B., B.Ch.Belf., D.P.M., Assistant Medical Officer, Claybury Mental Hospital, Woodford Bridge, Essex.

1929. Laws, John Joseph, M.R.C.S., L.R.C.P.Lond., D.P.M., Assistant Medical Officer, Horton Mental Hospital, Epsom.

1915. Leech, Henry Brougham, B.A., M.D., B.Ch.Dubl., Deputy Medical Superintendent, Warwick County Mental Hospital, Hatton. (Acting Registrar, 1923-24.)

1909. Leech, John Frederick Wolseley, B.A., M.D., B.Ch.Dubl., D.P.M., Assistant Medical Officer, Wilts County Mental Hospital, Devizes. 
1899. Leeper, Richard R., L.R.C.P., F.R.C.S.Irel., M.P.C., Medical Superintendent, St. Patrick's Hospital, Dublin. (Secretary, Irish Division, 1911-29, and Chairman, 1929-3I ; PRESIDENT, 1931-32.)

1906. Leggett, William, B.A., M.D., B.Ch.Dubl., Medical Officer, Smithston Mental Hospital, Greenock.

1927. Levinson, Reuben, M.B., Ch.B.Edin., D.P.H., D.P.M., Assistant Medical Officer, West Ham Mental Hospital, Goodmayes, Essex.

1930. Lewis, Edmund Oliver, M.A.Camb., D.Sc.Lond., M.R.C.S., L.R.C.P.Lond., Commissioner, Board of Control ; 34, Cholmeley Crescent, Highgate, N. 6.

1916. Lewis, Edward, F.R.F.P.S.Glasg., L.R.C.P.\&S.Edin., Medical Superintendent, Hensol Castle Institution, Pontyclun, Glamorgan.

1924. Lewis, John Biddulph Strafford, M.A.Camb., M.R.C.S., L.R.C.P.Lond., D.P.M., Deputy Medical Superintendent, Banstead Mental Hospital, Sutton, Surrey.

1931. Liddell, John Kenneth Crawford, M.B., Ch.B.Edin., Senior Assistant Medical Officer, Barnwood House, Gloucester.

1920. Lilly, George Austen, M.C., M.A., M.D.Camb., M.R.C.S., L.R.C.P.Lond., D.P.M., Medical Superintendent, Cane Hill Mental Hospital, Coulsdon, Surrey.

1927. Lindsay, Thomas, M.D., F.R.C.S.Edin., D.P.M., Medical Superintendent, Caterham Mental Hospital; The Pines, Caterham, Surrey.

1908. Litteljohn, Edward Salterne, M.R.C.S., L.R.C.P.Lond., Medical Superintendent, Manor Certified Institution, Epsom.

1925. Littlejohn, Mary Victoria, M.B., Ch.B.Aberd., D.P.M., 16, Northgate Street, Warwick.

192 I. Livesay, Arthur William Bligh, M.B., C.M., F.R.C.S.Edin., Assistant Medical Officer, St. Andrew's Hospital, Thorpe, Norwich.

1922. Logan, Frederick Colquhoun, M.B., Ch.B., F.R.F.P.S.Glasg., Medical Superintendent, County and City Mental Hospital, Gloucester ; Lindi, Horton Road, Gloucester.

1932. Logan, William Robert, M.B., Ch.B., Assistant Physician, Royal Mental Hospital, Aberdeen.

1931. Lord, Mrs. Ruby Thornton (née Carr), M.B., Ch.B.Edin., D.P.M., Assistant Medical Officer, County and City Mental Hospital, Littlemore, Oxford.

1924. Lornie, Peter, M.D., Ch.B.Edin., Senior Assistant Medical Officer, Monmouth County Mental Hospital, Abergavenny.

1924. Lothian, Douglas B. M., M.B., Ch.B., M.R.C.P.Edin., D.P.M., Deputy Medical Superintendent, Royal Scottish National Institution, Larbert, Stirlingshire.

1929. Love, Gwyneth Duncan, M.B., Ch.B.Edin., Assistant Physician, Bootham Park, York.

1923. Lovell, Clement, M.D., B.S.Lond., Pathologist, Bethlem Royal Hospital, Monk's Orchard, Beckenham, Kent; Beach Road, Emsworth, Hants.

1931. Lowenfeld, Margaret Frances Jane, M.R.C.S.Eng., L.R.C.P.Lond., Hon. Medical Director, The Children's Clinic for the Treatment and Study of Nervous and Delicate Children ; 2I, Devonshire Place, London, W. r.

1906. Lowry, James Arthur, M.D., B.Ch.R.U.I., Medical Superintendent, Surrey County Mental Hospital, Brookwood.

1928. Lowson, William, M.B., Ch.B.St. Andr., Medical Officer, Moat House, Tamworth ; 9, Colehill, Tamworth, Staffordshire.

1926. Lucas, Edmund Stanley Sayer, M.R.C.S., L.R.C.P.Lond., I.M.S., c/o Grindlay \& Co., Bombay, India.

1929. Luff, Mary Constance, M.B., B.S., M.R.C.S., L.R.C.P.Lond., D.P.M., Physician, The Institute of Medical Psychology ; 35, Weymouth Street, London, W. I.

1930. Lyons, Bernard, M.B., B.Ch., B.A.O.N.U.I., Assistant Medical Officer, District Mental Hospital, Enniscorthy, co. Wexford.

1920. McAlister, William Malcolm, M.A., M.B., Ch.B., F.R.C.P.Edin., Dipl. Psych., Medical Superintendent, Bangour Village, West Lothian. (Lect. on Psychiat., Univ. of Edin.)

1906. Macarthur, John, M.R.C.S., L.R.C.P.Lond., D.P.M., Medical Superintendent, County Mental Hospital, Bracebridge Heath, Lincs.

193r. MacArthur, Mary Elizabeth, M.B., B.Ch.N.U.I., Assistant Medical Officer, The Retreat, York.

1923. Macaulay, Douglas Ian Otto, M.D., Ch.B.Edin., D.P.M., Medical Superintendent, Chiswick House, Pinner, Middlesex.

c 
1880. MacBryan, Henry Crawford, L.R.C.P.\&S.Edin., Medical Superintendent, Kingsdown House, Box, Wilts.

1926. MacCallum, Alexander Grigor, M.B., Ch.B.Glasg., I5, Woodbourne Avenue, Streatham, London, S.W. 16.

1929. Maccallum, Archibald Montgomery, M.B., Ch.B.Glasg., Assistant Medical Officer, Ipswich Mental Hospital, Ipswich.

1929. MacCalman, Douglas Robert, M.B., Ch.B.Glasg., Alderdale, Carr Bridge, Invernessshire.

1929. McCartan, William, M.D.Belf., D.P.H., D.P.M., Assistant Medical Officer, West Park Mental Hospital, Epsom.

1900. McClintock, John, L.R.C.P.\&S.Edin., Resident Medical Superintendent, Grove House, Church Stretton, Salop.

1931. McConnell, Joyce, M.B., B.S.Lond., D.P.M., Assistant Medical Officer, Long Grove Mental Hospital, Epsom.

1927. McCoull, George, M.B., B.S.Durh., L.R.C.P.\&.S.Edin., L.R.F.P.S.Glasg., Medical Officer, Prudhoe Hall Colony for Mental Defectives; Tyne View, Prudhoe-on-Tyne.

1920. McCowan, Peter Knight, M.D., Ch.B.Edin., M.R.C.P.Lond., D.P.M., Barrister-at-Law ; Medical Superintendent, Cardiff City Mental Hospital, Whitchurch. (Lect. in Ment. Dis., Welsh National School of Medicine.) (Secretary, Research and Clinical Committee, since 1931.)

1932. McCullev, William John, B.Sc., M.B., Ch.B., D.P.M., Assistant Medical Officer, City Mental Hospital, Mapperley Hill, Nottingham.

1921. McCutcheon, Archibald Munn, M.B., Ch.B., F.R.F.P.S.Glasg., Medical Superintendent, Monyhull Colony, King's Heath, Birmingham.

1931. McDiarmid, Neil, M.B., Ch.B.Glasg., Assistant Medical Officer, Lancashire County Mental Hospital, Whittingham, Preston.

1901. MacDonald, James Hogg, M.B., Ch.B., F.R.F.P.S.Glasg., Medical Superintendent, Glasgow District Asylum, Hawkhead, Cardonald, Glasgow. (Lect. on Psychol. Med., Univ. of Glasgow.) (Chairman, Scottish Division, since 1932.)

1884. MacDonald, P. W., M.D., C.M.Aberd., J.P., Grasmere, Radipole, Weymouth. (First Secretary, S.W. Division, 1894-1905; PRESIDENT, 1907-8.)

I9I I. MacDonald, Ranald, O.B.E., M.D., Ch.B.Edin., D.P.M., Medical Superintendent, Coton Hill Mental Hospital, Stafford.

1928. Macdonald, Robert William, M.B., Ch.B.Glasg., Medical Officer, Ministry of Pensions, Queen Alexandra Hospital, Cosham, Hampshire.

1905. MacDonald, William Fraser, M.B., Ch.B.Edin., M.P.C., Olive Lodge, Polworth Terrace, Edinburgh.

1931. MacDougall, Donald John, M.B., Ch.B., Major, R.A.M.C., Officer-in-Charge, " $\mathrm{D}$ " Block, Royal Victoria Hospital, Netley.

1928. McDougall, John, M.B., Ch.B.Glasg., Senior Assistant Physician, Crichton Royal, Dumfries.

1906. McDowall, Colin Francis Frederick, M.D., B.S.Durh., M.R.C.S., L.R.C.P.Lond., Medical Superintendent, Ticehurst House, Ticehurst, Sussex. (Secretary, Library Committee, since 1922.)

1931. McElroy, William Ernest, M.B., Ch.B., B.A.O., Assistant Medical Officer, Park Prewett Mental Hospital, Basingstoke.

1895. Macfarlane, Neil M., C.B.E., M.D., C.M.Aberd., 565, West Street, Durban, Natal, South Africa.

1924. Macfarlane, Robert Melvin, M.B., Ch.B.Edin., D.P.H., D.P.M., Assistant Medical Officer, West Ham Mental Hospital, Goodmayes, Essex.

1923. McGarvey, John, M.B., B.Ch.Belf., D.P.M., Medical Superintendent, Somerset and Bath Mental Hospital, Wells.

1922. McGeorge, Margaret Turner, M.B., Ch.B.Glasg., 35, Glenesk Road, Eltham, S.E. 9.

1928. MacGilp, Finlay Duncan, M.B., Ch.B.St. Andr., Assistant Medical Officer, Durham County Mental Hospital, Winterton, Ferry Hill, co. Durham.

1925. McGlashan, William Reid, M.A., M.B., Ch.B.Aberd., D.P.M.

1925. MacGowan, Agnes Mildred, M.B., Ch.B.Edin., D.P.M., Assistant Medical Officer and Pathologist, Bangour Village, Uphall, Linlithgowshire.

1929. McGrath, Annie Margaret, B.C., M.R.C.S., L.R.C.P., Pathologist, Herts County Mental Hospital, Hill End, St. Albans.

I92I. McGrath, Mathew Joseph, M.B., B.Ch.R.U.I., D.P.M., Deputy Medical Superintendent, West Riding Mental Hospital; Gortmore, Bar Lane, Stanley, near Wakefield. 
1902. McGregor, John, M.B., Ch.B.Edin., Deputy Medical Superintendent, Glamorgan County Mental Hospital, Bridgend.

1924. McInnes, John, M.B., Ch.B.Glasg., D.P.M., Assistant Medical Officer, City Mental Hospital, Willerby, Hull.

1932. McInnes, Robert Gow, L.R.C.P.\&S.Edin., M.R.C.P.Edin., Senior Assistant Physician, Royal Edinburgh Hospital; West House, Morningside, Edinburgh.

1921. McKail, Robert Buchanan Forbes, M.B., Ch.B.Glasg., D.P.M., Deputy Medical Superintendent, Calderstones Certified Institution, Whalley, near Blackburn.

1924. Mackay, George William John, M.B., Ch.B.Edin., D.P.M., Medical Officer, Somerset Mental Deficiency Committee; " Beaford", Greenway Road, Taunton, Somerset.

1929. Mackay, John, M.B., Ch.B.Glasg., Assistant Medical Officer, Cheadle Royal, Cheadle, Cheshire.

1914. Mackay, Magnus Ross, M.C., M.B., Ch.B.Edin., Medical Superintendent, Newport Borough Mental Hospital, Caerleon, Mon.

I89 I. Mackenzie, Henry James, M.B., C.M.Edin., M.P.C., 254, Bishopsthorpe Road, York.

1927. Mackenzie, Ivy, M.A., B.Sc., M.D., F.R.F.P.S.Glasg., Consulting Physician, Glasgow District Board of Control ; ro, Woodside Terrace, Glasgow, C. 3.

I9I I. Mackenzie, John Cosserat, M.B., Ch.B.Edin., Assistant Medical Officer, Stafford Mental Hospital, Burntwood, Lichfield.

1926. Mackenzie, John Muir, M.B., Ch.B.Glasg., D.P.M., Assistant Medical Officer, Hollvmoor Mental Hospital, Northfield, Birmingham.

1927. Mackenzie, Murdo, M.B., B.S., M.R.C.P.Lond., Senior Assistant Physician, Bethlem Royal Hospital, Monk's Orchard, Beckenham, Kent.

1930. Mackenzie, Myra, M.B., Ch.B.Aberd., Assistant Medical Officer, The Lawn, Lincoln.

1903. Mackenzie, Theodore Charles, M.D., Ch.B., F.R.C.P.Edin., M.P.C., Druim, Inverness.

1921. Mackie, George, D.S.O., M.D., Ch.B.Edin., Thornyhill, Abbey Road, Great Malvern.

1931. McLaughlin, Francis Leo, M.D., B.Ch.N.U.I., Assistant Medical Officer, Colney Hatch Mental Hospital, New Southgate, N. rr.

1927. Macleod, John, M.B., Ch.B.Edin., Assistant Medical Officer, Glasgow District Mental Hospital, Woodilee, Lenzie, N.B.

1921. Macleod, Neil, M.D.Edin., D.P.M., Medical Superintendent, The Retreat, York.

1925. McManus, Hugh Charles, M.B., Ch.B.Vict., D.P.M., The Hall, Wedmore, Somerset.

1930. McMenamin, Francis de Sales, M.C., M.B., Ch.B.Edin., 38, Lower Baggot Street, Dublin.

1931. Macmillan, Donald MacPhail, M.B., Ch.B.Glasg., D.P.M., Medical Superintendent, Great Barr Park Colony, near Birmingham.

1929. Macmillan, Duncan, B.Sc., M.D., M.R.C.P.Edin., D.Psych., Deputy Medical Superintendent, Nottingham City Mental Hospital; Robinwood, Wells Road, Mapperley, Nottingham.

1904. Macnamara, Eric Danvers, M.A., M.D., B.Ch.Camb., F.R.C.P.Lond., 87, Harley Street, London, W. I.

1925. MacNiven, Angus, M.B., Ch.B.Glasg., M.R.C.P.Edin., D.P.M., Physician Superintendent, Glasgow Royal Mental Hospital ; 2, Whittingehame Gardens, Glasgow, W. 2.

19 10. MacPhail, Hector Duncan, O.B.E., M.A., M.D., Ch.B.Edin., Medical Superintendent, City Mental Hospital, Gosforth, Newcastle-upon-Tyne. (Lect. on Psychol. Med., Univ. of Durh.)

1922. Macphail, Iain Ross, L.R.C.P.\&S.Edin., L.R.F.P.S.Glasg., Hartwood, Lanarkshire.

19or. McRae, Douglas, M.D., C.M., F.R.C.P.Edin., J.P., Physician-Superintendent, Glengall Hospital ; Glengall House, Ayr, N.B. (Assistant Editor, 1915-20, and Co-Editor of Journal since 1920; Chairman, Scottish Division, 1931-32.)

1922. McWilliam, William, M.D., Ch.B., F.R.F.P.S.Glasg., D.P.M., Medical Superintendent, District Asylum, Inverness.

1925. Madgwick, John Reginald Alexander, M.B., B.S., M.R.C.S., L.R.C.P.Lond., D.P.M., Assistant Medical Officer, Long Grove Mental Hospital, Epsom, Surrey.

1923. Madill, Joseph Thomas Herbert, B.A.N.U.I., M.B., B.Ch.Edin., F.R.F.P.S.Glasg., D.P.M., M.P.C., Medical Superintendent, Cumberland and Westmorland Mental Hospital, Carlisle.

1931. Magrath, Donald, M.B., Ch.B.Birm., M.R.C.S., L.R.C.P.Lond., D.P.H., Assistant Medical Officer, Caterham Mental Hospital, Caterham, Surrev.

1929. Mahony, Elizabeth Maud, M.B., Ch.B., B.A.O.N.U.I., Assistant Medical Officer, Mental Hospital, Monaghan, Ireland.

1928. Main, Dorothy Mary, M.B., Ch.B.Glasg., Assistant Medical Officer, City Mental Hospital, Willerby, Hull. 
1931. Malloy, Joseph Holder, M.D.St. And., Assistant Medical Officer, Staffordshire County Mental Hospital, Cheddleton.

1908. Mapother, Edward, M.D., B.S.Lond., F.R.C.P.Lond., F.R.C.S.Eng., Medical Superin. tendent, The Maudsley Hospital, Denmark Hill, London, S.E. 5 ; 19, Queen Anne Street, London, W. I. (Lect. in Psych. Med., King's Coll. Hosp.)

1903. Marnan, John, B.A., M.B., B.Ch.Dubl., 144, London Road, Gloucester.

1896. Marr, Hamilton, M.D., C.M., F.R.F.P.S.Glasg., M.P.C., Commissioner, General Board of Control for Scotland; 1o, Succoth Avenue, Edinburgh. (Secretary, Scottish Division, 1907-10; President, 1927-28.)

1929. Marsh, Rex Godfrey Blake, M.R.C.S., L.R.C.P.Lond., D.P.M., Deputy Medical Superintendent, Leavesden Mental Hospital; Gabled House, Woodside, near Watford, Herts.

1926. Martin, Alexander Reid, M.B., B.Ch.Belf., D.P.M., Assistant Physician, Sheppard and Pratt Hospital, Townson, Baltimore, Md., U.S.A.

1922. Martin, Frederick Robertson, M.D., Ch.B.Glasg., D.P.M., 6, Grove Road, Sutton, Surrey.

I896. Martin, James Charles, L.R.C.S.\&.P.Irel., J.P., Medical Superintendent, District Mental Hospital, Letterkenny, Donegal.

1930. Martin, John James Black, M.A., M.D., B.Ch., M.R.C.P., D.P.M., Deputy Medical Superintendent, Dorset County Mental Hospital, Herrison, Dorchester.

1907. Martin, Mary Edith, L.S.A., L.R.C.P.\&S.Edin., L.R.F.P.S.Glasg., M.P.C., II, The Drive, Hove, Sussex.

1914. Martin, Samuel Edgar, M.B., Ch.B.Edin., Barrister-at-Law, Medical Superintendent, The Old Manor, Salisbury. (Secretary, South-Western Division, since 1930.)

1921. Masefield, William Gordon, M.R.C.S., L.R.C.P.Lond., D.P.M., Medical Superintendent, Essex County Mental Hospital, Brentwood. (Secretary, Educational Committee, since 1930.$)$

19rI. Mathieson, James Moir, M.B., Ch.B.Aberd., Assistant Medical Officer, South Yorks Mental Hospital, Wadsley, Sheffield.

1932. Maxwell, Robert Warnock, M.R.C.S., L.R.C.P., Assistant Medical Officer, Somerset and Bath Mental Hospital, Wells.

1926. May, George Francis, M.D., C.M.McGill, L.M.S.S.A., Medical Superintendent, Durham County Mental Hospital, Winterton, Ferry Hill.

1932. Menzies, Archibald, L.R.C.P.\&S.Edin., L.R.F.P.S.Glasg., Assistant Medical Officer, Hawkhead Mental Hospital, Glasgow.

1926. Menzies, Duncan, M.A., M.B., Ch.B.Aberd., D.P.M., Deputy Medical Superintendent, Plymouth Mental Hospital, Ivybridge, Devon.

1890. Menzies, William F., B.Sc., M.D., C.M.Edin., F.R.C.P.Lond., Medical Superintendent Stafford County Mental Hospital, Cheddleton, Leek. (Vice-Chairman, Parliamentary Committee, since 1932 ; PRESIDENT, 1920-21.)

1877. Merson, John, M.A., M.D., C.M.Aberd., Willerby, Brayton Road, Selby.

1932. Methven, James Black, M.B., Ch.B.Edin., Assistant Medical Officer, Fife District Asylum, Cupar.

1910. Middlemiss, James Ernest, F.R.F.P.S.Glasg., M.R.C.S., L.R.C.P.Lond., M.P.C., “ The Crest," Scott Hall Road, Chapel-Allerton, Leeds.

1928. Mill, Laura Margaret Dorothea, M.B., Ch.B.Glasg., Public Health Office, 23, Montrose Street, Glasgow.

1929. Miller, Emanuel, M.A.Camb., M.R.C.S., L.R.C.P.Lond., D.P.M., Psychiatrist, East London Child Guidance Clinic; 28, Wimpole Street, London, W. 1 .

1930. Miller, Frederick Richard Lanfear, M.R.C.S., L.R.C.P.Lond., D.T.M.\&H., 33, Pembridge Villas, London, W. Ir.

1925. Miller, Josephine Alcorn Carson, L.R.C.P.\&S.Irel., Oxhey Grove, Hatch End, Middlesex.

1924. Miller, Robert Stewart, M.D., Ch.B.Glasg., Director, Khanka Mental Hospital, Cairo, Egypt.

1893. Mills, John, M.B., B.Ch.R.U.I., D.M.D., Medical Superintendent, District Mental Asylum, Ballinasloe, Ireland.

1931. Milmo, Dermod Hubert Francis, M.B., B.Ch.Dubl., D.P.H., Assistant Medical Officer, Caterham Mental Hospital, Caterham, Surrey.

1923. Minski, Louis, M.D., B.S.Durh., M.R.C.P.Lond., D.P.M., Assistant Medical Officer, Maudsley Hospital ; South Lodge, Champion Hill, London, S.E. 5.

1922. Molony, Charles Bernard, M.B., Ch.B.N.U.I., Assistant Medical Officer, District Mental Hospital, Limerick. 
1910. Monnington, Richard Caldicott, M.D., Ch.B.Edin., D.P.H., D.P.M., Neurologist, Ministry of Pensions; 33, New Street, Salisbury.

1915. Monrad-Krohn, G. H., B.A., M.D., B.S.Oslo, F.R.C.P.Lond., M.R.C.S.Eng., M.P.C., Rikshospitalet, Oslo, Norway. (Prof. of Med., Royal Frederick University, Oslo.)

1931. Moodie, William, M.D., M.R.C.P.Lond., D.P.M., Medical Director, London Child Guidance Clinic, Canonbury, N. I ; I8, New Cavendish Street, London, W. r.

1925. Moran, Patrick, M.B., B.Ch.N.U.I., D.P.M., Assistant Medical Officer, District Mental Hospital, Mullingar.

1917. Morris, Bedlington Howel, M.B., B.S.Durh., Inspector-General of Hospitals, South Australia ; "Tros-y-Parc ", Pembroke Street, St. Peter's, Adelaide, South Australia.

1925. Morris, John Vincent, B.A., M.B., B.Ch.Dubl., Assistant Medical Officer, St. Andrew's Hospital, Thorpe, Norwich.

1896. Mould, Gilbert Edward, M.R.C.S., L.R.C.P.Lond., The Grange, Rotherham, Yorks

1914. Moyes, John Murray, M.B., Ch.B.Edin., D.P.M., Tue Brook Villa, Liverpool, E.

1919. Mules, Annie Shortridge, M.R.C.S., L.R.C.P.Lond., Court Hall, Kenton, Douth Devon.

1907. Mules, Bertha Mary, M.D., B.S.Durh., Court Hall, Kenton, South Devon.

1929. Mullin, Bartholomew Joseph, M.C., L.R.C.P.\&S.Irel., D.P.H., D.P.M., Deputy Medical Superintendent, Wonford House Hospital, Exeter.

1929. Munro, Thomas Arthur, M.B., Ch.B., M.R.C.P. Edin., Dipl. Psych., Assistant Physician, Phipp's Psychiatric Clinic, Baltimore, U.S.A.; 2o, Merchiston Gardens, Edinburgh.

1925. Murdoch, James Wilson, M.B., Ch.B.Aberd., Senior Assistant Medical Superintendent, Central Mental Hospital, Tanjong Rambutan, Perak, Federaced Malay States.

1931. Murray, Helen Sara Euphemia, M.B., Ch.B.Edin., Assistant Medical Officer, Lancashire County Mental Hospital, Whittingham, Preston.

1932. Murray, John Raymund, M.B., B.S.Lond., M.R.C.S., L.R.C.P., Assistant Medical Officer, Claybury Mental Hospital, Woodford Bridge, Essex.

1929. Muthiah, Asainayagam Richard, L.M.S.Singapore, Assistant Physician, Central Mental Hospital, Tanjong Rambutan, Perak, Federated Malay States.

1903. Navarra, Norman, M.R.C.S., L.R.C.P.Lond., D.P.M., Deputy Medical Superintendent, City of London Mental Hospital, Stone, Dartford.

1910. Neill, Alex. William, M.D., Ch.B.Edin., Physician-Superintendent, The Warneford Hospital, Oxford.

1920. Nicol, William Drew, M.B., B.S., M.R.C.P.Lond., D.P.M., Medical Superintendent, Horton Mental Hospital, Epsom. (Lect. on Psychiatry, London School of Medicine for Women.)

1923. Nicole, J. Ernest, L.M.S.S.A., D.P.M., Senior Assistant Medical Officer, Lancashire County Mental Hospital, Winwick, Warrington.

1923. Nicoll, James, M.D., C.M.Edin., D.P.H., Medical Superintendent, Fountain Mental Hospital, Tooting Grove, London, S.W. I 7.

1920. Nix, Sidney, M.D., B.S.Durh., L.R.C.P.\&S.Edin., L.R.F.P.S.Glasg., Deputy Medical Superintendent, Graylingwell Mental Hospital, Chichester.

1922. Noble, Ralph Athelstane, M.B., Ch.M.Syd., D.P.M., ro, Edgehill Terrace, New Haven, Connecticut, U.S.A.

1909. Norman, Hubert James, M.B., Ch.B.Edin., D.P.H., Medical Superintendent, Camberwell House Mental Hospital, London, S.E. 5 ; Northcotes, 79, West Hill, Sydenham, London, S.E. 26. (Lect. on Ment. Dis., Westm. Hosp.)

1923. Noronha, Frank, M.B., C.M.Madras, D.P.M., Superintendent, Mental Hospital, Bangalore

City, India.
1932. Norris, Frank Edwin, M.R.C.S.Eng., L.R.C.P.Lond., Assistant Medical Officer, The Coppice, Nottingham.

1926. Northcote, Muriel L. M., M.B., B.S., M.R.C.S., L.R.C.P.Lond., D.P.M., Deputy Medical Superintendent, City Mental Hospital, Whitchurch, Cardiff.

1930. O'Brien, Eveleen J., M.B., B.Ch., B.A.O.N.U.I., D.P.M., Assistant Medical Officer, Portrane Mental Hospital, Donabate, co. Dublin.

1929. O'Connell, Daniel Joseph, M.B., B.Ch.N.U.I., D.P.M., Assistant Medical Officer, St. Andrew's Hospital, Northampton.

1924. Odlum, Doris Maude, M.A.Oxon., B.A.Lond., M.R.C.S., L.R.C.P.Lond., D.P.M., 42, Harley Street, London, W. I.

1918. Ogilvie, William Mitchell, M.B., C.M.Aberd., “Monymusk”, 2, Riselaw Terrace, Edinburgh. 
I9or. Ogilvy, David, B.A., M.D., B.Ch.Dubl., Medical Superintendent, Long Grove Mental Hospital, Epsom.

1911. Oliver, Norman Henry, M.R.C.S., L.R.C.P.Lond., Barrister-at-Law, Medical Superintendent, Ministry of Pensions Hospital; Latchmere, Ham Common, Surrey.

1922. O'Flaherty, Very Rev. Claude, M.B., Ch.B.Edin., The College, Millport, Buteshire.

1920. O'Neill, Arthur, O.B.E., M.R.C.S., L.R.C.P.Lond., Medical Superintendent, Napsbury Mental Hospital, St. Albans, Herts.

1924. O'Reilly, James Joseph, M.B., B.Ch.Belf., D.P.M., Deputy Medical Superintendent, City Mental Hospital, Winson Green, Birmingham.

1930. O'Riordan, John Joseph, M.B., Ch.B., D.P.M., Assistant Medical Officer, North Riding Mental Hospital, York.

1902. Orr, David, M.D., C.M.Edin., M.P.C., 24, Grange Road, Edinburgh.

r910. Orr, James Henry Cubitt, M.D., Ch.B.Edin., Medical Superintendent, Midlothian and Peebles Asylum, Rosslyn Castle.

1932. O'Sullivan, Edward N. M., B.A., M.B., B.Ch., B.A.O., D.P.M.N.U.I., Assistant Medical Officer, District Mental Hospital, Killarney.

1916. Overbeck-Wright, Alexander William, M.D., Ch.B.Aberd., D.P.H., M.P.C., Lt.-Col. I.M.S., Superintendent, Asylum House, Agra, U.P., India ; c/o Messrs. King, King \& Co., Bombay, India.

1928. Paddle, Kenneth Cecil Laurence, M.C., M.R.C.S., L.R.C.P.Lond., D.P.M., Deputy Medical Superintendent, Caterham Mental Hospital, Caterham, Surrey.

1930. Page, William Robert, B.A., M.B., Ch.M.Sydney, D.P.M.Lond., Hon. Psychiatrist Sydney Hospital and St. Vincent's Hospital, Sydney; 221, Macquarie Street, Sydney, N.S.W., Australia.

1927. Pal, Sachindra Bhushan, B.A., L.M.S.Calc., Senior Assistant Physician, Central Mental Hospital, Tanjong Rambutan, Federated Malay States.

1927. Parasuram, Govindarajpuram Rampattar, B.A., L.M.S.Madras, M.R.C.P.Edin., Deputy Medical Superintendent, Government Mental Hospital, Madras.

1932. Parfitt, David Neil, M.B., B.S.Lond., B.Sc., D.P.M., M.R.C.S., L.R.C.P., Senior Assistant Medical Officer, Warwick County Mental Hospital, Hatton.

1931. Parker, William Patrick Hugh, L.R.C.P.\&S.I., Assistant Medical Superintendent, Mental Hospital, Toowoomba, Queensland, Australia.

1920. Parkin, George Gray, M.D., Ch.B.Vict., Deputy Medical Superintendent, Cheshire County Mental Hospital, Parkside, Macclesfield.

1920. Parnis, Henry William, B.Sc., M.D.Malta, M.R.C.S., L.R.C.P.Lond., D.P.M., Deputy Medical Superintendent, Bexley Mental Hospital, Bexley, Kent.

1916. Patch, Charles James Lodge, M.C., L.R.C.P.\&S.Edin., L.R.F.P.S.Glasg., Capt. I.M.S., 39, Jail Road, Lahore, India.

1929. Paterson, Arthur Spencer, B.A.Oxon., M.B., Ch.B., M.R.C.P.Edin., Research Fellow, The Maudsley Hospital; 76, Wimpole Street, London, W. I.

1930. Paton, Thomas, M.B., Ch.B.Glasg., D.P.M., Assistant Medical Officer, Brookwood Mental Hospital, Surrey.

1899. Patrick, John, M.B., B.Ch.R.U.I., Medical Superintendent, District Asylum, Omagh.

1928. Patterson, Edward Cecil, M.B., B.Ch.Belf., Assistant Medical Officer, Devon Mental Hospital, Exminster.

1931. Paul, Arthur Blackwell, M.A., M.B., B.Ch.Camb., Consulting Physician, Cheshire County Mental Hospital, Chester; Hon. Physician, Chester Royal Infirmary ; 22, Nicholas Street, Chester.

1929. Pearce, John Dalziel Wyndham, M.A., M.B., Ch.B.Edin., Assistant Medical Officer, City Mental Hospital, Leicester.

1920. Pearn, Oscar Phillips Napier, L.S.A., M.R.C.S., L.R.C.P.Lond., D.P.M., Deputy Medical Superintendent, Cane Hill Mental Hospital, Coulsdon, Surrey.

1931. Pearson, Kate Young, M.B., Ch.B., Assistant Medical Officer, Rampton State Institution, Retford, Notts.

1913. Penny, Robert Augustus Greenwood, M.R.C.S., L.R.C.P.Lond., Assistant Medical Officer, Devon County Mental Hospital, Exminster.

1927. Perera, Clement Osmund, M.R.C.S., L.R.C.P.Lond., D.P.M., Assistant Medical Superintendent, Mental Hospital, Angoda, Ceylon.

1928. Perk, David, M.D.Leeds, D.P.M., Assistant Medical Officer, West Riding Mental Hospital Menston, near Leeds.

1929. Peters, Gordon Frank, M.B., B.S., M.R.C.S., L.R.C.P.Lond., D.P.M., Deputy Medical Superintendent, Claybury Mental Hospital, Woodford Bridge, Essex. 
1911. Petrie, Alfred Alexander Webster, M.D., B.S., F.R.C.P.Lond,, M.D., F.R.C.S.Edin., D.P.M., Medical Superintendent, Banstead Mental Hospital, Sutton, Surrey. (Lect. on Ment. Dis., Charing Cross Hosp. and West Lond. Post.-Grad. College.) (Vice Chairman, Research and Clinical Committee, since 1932.)

1908. Phillips, John George Porter, M.D., B.S., F.R.C.P.Lond., M.P.C., Kesident PhysicianSuperintendent, Bethlem Royal Hospital, Monk's Orchard, Beckenham, Kent ; 19, Cavendish Square, London, W. I. (Lect. on Ment. Dis., St. Bart.'s Hosp.) (Secretary, Educational Committee, I9I 3-20.)

1906. Phillips, Nathaniel Richard, M.D.Brux., M.R.C.S., L.R.C.P.Lond., D.P.M., Medical Superintendent, Monmouth County Mental Hospital, Abergavenny.

1905. Phillips, Norman Routh, M.D.Brux., M.R.C.S., L.R.C.P.Lond., D.P.M., Deputy Medical Superintendent, St. Andrew's Hospital, Northampton.

1921. Phillips, Philip Gordon, L.R.C.P.\&S.Edin., L.R.F.P.S.Glasg., Bryn, The Avenue, Collingham, Leeds.

1924. Pickworth, Frederick Alfred, B.Sc., M.B., B.S., M.R.C.S., L.R.C.P.Lond., A.I.C.(exam.), Ph.C., Director, Joint Board of Research for Mental Diseases, City and University of Birmingham; Hollymoor Mental Hospital, Northfield, Birmingham.

I 888. Pietersen, James F. G., M.R.C.S., L.R.C.P.Lond., Ashwood House, Kingswinford, near Dudley, Stafford.

1 896. Planck, Charles, M.A.Camb., M.R.C.S., L.R.C.P.Lond., “Pontresina”, Perrymount Road, Haywards Heath, Sussex.

I91 3. Potts, William A., M.A.Camb., M.D.Edin.\&Birm., M.R.C.S., I..R.C.P.Lond., Medical Officer, Birmingham Committee for the Care of the Feeble-Minded; I 8 , Hagley Road, Edgbaston, Birmingham.

1923. Power, Thomas Declan, B.A., M.D., B.Ch.Dubl., M.R.C.P.Lond., D.P.H., D.P.M., Deputy Medical Superintendent, Essex County Mental Hospital, Brentwood.

1921. Poynder, Ernest George Thornton, M.R.C.S., L.R.C.P.Lond., D.P.M., Medical Superintendent, Plymouth Mental Hospital, Blackadon, Ivybridge, Devon.

1931. Prentice, David, M.B., Ch.B.Glasg., Assistant Medical Officer, Lancashire County Mental Hospital, Whittingham, Preston.

1918. Prideaux, Joseph Francis Engledue, M.R.C.S., L.R.C.P.Lond., D.C.M.S. ; Ministry of Pensions, I, Sanctuary Buildings, Great Smith Street, London, S.W. I.

1928. Quine, Margaret Annette, M.B., B.S., M.R.C.S., L.R.C.P.Lond., D.P.M., Assistant Medical Officer, Lancashire County Mental Hospital, Winwick, Warrington.

1930. Rae, James Burnett, M.B., Ch.B.Aberd., Hon. Physician in Charge, Department of Psychological Medicine, Croydon General Hospital ; 93, Harley Street, London, W. I.

1928. Raitt, William John, M.B., Ch.B.Aberd., Dipl. Psych., Assistant Physician, Crichton Royal, Dumfries.

1894. Rambaut, Daniel F., M.A., M.D., B.Ch.Dubl., Medical Superintendent, St. Andrew's Hospital ; Priory Cottage, Northampton. (Registrar since 1924.)

1889. Raw, Nathan, C.M.G., M.D., B.S., M.R.C.P.Lond., F.R.C.S., F.R.S.Edin., L.S.Sc.Durh., M.P.C., Lord Chancellor's Visitor; 3o, Clarendon Court, Maida Vale, London, W. 9. (Chairman, Parliamentary Committee since 1926; President, 1929-30.)

191 3. Read, Charles Stanford, M.D., M.R.C.S., L.R.C.P.Lond., 62, Queen Anne Street, London, W. I. (Lect. on Psychol. Med., Bethlem Royal Hospital.)

1920. Read, Walter Woolfe, M.D.Brux., M.R.C.S., L.R.C.P.Lond., Medical Superintendent, Berks County Mental Hospital, Moulsford, Wallingford.

1930. Rees, John Rawlings, M.A., M.D.Camb., D.P.H., Deputy Director, The Institute of Medical Psychology; I4, Wimpole Street, London, W. I.

1927. Rees, Thomas Percy, B.Sc., M.B., B.Ch.Wales, M.R.C.P.Lond., M.R.C.S.Eng., D.P.M., Senior Assistant Medical Officer, Croydon Mental Hospital, Upper Warlingham, Surrey.

I9I I. Reeve, Ernest Frederick, M.B., B.S., M.R.C.S., L.R.C.P.Lond., Medical Superintendent, Lancashire County Mental Hospital, Rainhill, near Liverpool. (Lect. on Ment. Dis., Univ. of Liverp.)

1931. Reid, Benjamin, M.B., Ch.B.Glasg., D.P.M., Senior Medical Officer, Lancashire County Mental Hospital, Whittingham, Preston.

1911. Reid, Daniel McKinley, M.D., Ch.B., F.R.F.P.S.Glasg., Medical Superintendent, City Mental Hospital, Exeter. 
1910. Reid, William, M.A.St.And., M.B., Ch.B.Edin., Medical Superintendent, Stafford Mental Hospital, Burntwood, Lichfield.

1932. Reid, William Muirhead, L.R.C.P.\&S.Edin., L.R.F.P.S.Glasg., D.P.H., Assistant Physician, Royal Mental Hospital, Aberdeen.

1923. Retallack-Moloney, Herbert Thomas, M.R.C.S., L.R.C.P.Lond., D.P.M., Assistant Medical Officer, Hanwell Mental Hospital, Southall, Middlesex.

1899. Rice, David, M.D.Brux., M.R.C.S., L.R.C.P.Lond., D.P.H., Medical Superintendent, City Mental Hospital, Hellesdon, Norwich.

1929. Rich, Gilbert J., A.B., A.M., Ph.D.Cornell, M.D.Chicago, D.N.B., Institute for Juvenile Research, 907, South Lincoln Street, Chicago, U.S.A.

1897. Richard, William John, M.A., M.B., C.M., F.R.F.P.S.Glasg., Medical Superintendent, South General Hospital; Merryflats House, Govan, Glasgow.

1922. Riches, Reginald George, M.R.C.S., L.R.C.P.Lond., D.P.M., Deputy Medical Superintendent, Hanwell Mental Hospital, Southall, Middlesex.

1920. Rickman, John, M.A., M.D., B.Ch.Camb., Physician, London Clinic of Psycho-Analysis; II, Kent Terrace, London, N.W. r.

1931. Rivers, William Gregory, M.B., Ch.B.Edin., Medical Superintendent, Cornwall Mental Hospital, Bodmin.

1931. Roachsmith, C. E., Assistant Medical Officer, Napsbury Mental Hospital, St. Albans.

19rI. Robarts, Henry Howard, M.D., Ch.B.Edin., D.P.H., Medical Officer, East Lothian Mental Hospital, Ennerdale, Haddington, N.B.

1922. Robb, John Robert Beith, L.R.C.P.\&S.Edin., F.R.F.P.S.Glasg., Deputy Medical Superintendent, Glasgow District Mental Hospital, Gartloch, Gartcosh, N.B.

1921. Roberts, Edward Douglas Thomas, M.R.C.S., L.R.C.P.Lond., D.P.M., Assistant Medical Officer, Herts County Mental Hospital, Hill End, St. Albans.

1903. Roberts, Norcliffe, O.B.E., M.D., B.S.Durh., D.P.M., Medical Superintendent, West Park Mental Hospital, Epsom.

1927. Robertson, David, M.D., Ch.B.Glasg., Junior Assistant Physician, Bethlem Royal Hospital, Monk's Orchard, Beckenham, Kent.

1908. Robertson, George Dunlop, L.R.C.P.\&S.Edin., L.R.F.P.S.Glasg., Dipl. Psych., Hartwoodhill House, Hartwood, Lanarkshire.

1929. Robertson, James Andrew, M.B., Ch.B.Edin., 144, George Street, Hull.

1920. Robinson, William, M.D., Ch.B.Leeds, D.P.M., Medical Superintendent, City of London Mental Hospital, Stone, Dartford.

1922. Rodger, Kenneth Mann, M.B., Ch.B.Glasg., D.P.M., Deputy Medical Superintendent, Salop County Mental Hospital, Bicton Heath.

1914. Rodger, Murdoch Mann, M.D., Ch.B.Glasg., Dechmont, Helouan, Egypt.

1930. Rodger, Thomas Ferguson, B.Sc., M.B., Ch.B.Glasg., 43, Burnbank Terrace, Glasgow, N.W.

1908. Rodgers, Frederick Millar, O.B.E., M.D., Ch.B.Vict., D.P.H., Medical Superintendent, Lancashire County Mental Hospital, Winwick, Warrington.

1895. Rolleston, Lancelot William, C.B.E., M.B., B.S.Durh., M.R.C.S., L.R.C.P.Lond., Queen Anne's Mansions, St. James's Park, London, S.W. r.

1922. Rollins, Ernest Edward, B.A., M.B., B.Ch.Dubl., Resident Medical Officer, Brooke House, Upper Clapton, London, E. 5.

1931. Roper, William Francis, M.B., B.S.Lond., M.R.C.S., L.R.C.P., Medical Officer, H.M Prison, Liverpool; 21, Walton Park, Liverpool.

1888. Ross, Chisholm, M.D.Syd., M.B., C.M.Edin., 225, Macquarie Street, Sydney, New South Wales.

19 10. Ross, Donald, M.B., Ch.B., M.R.C.P.Edin., M.P.C., J.P., Medical Superintendent, Argyll and Bute Asylum; Tigh-na-Linne, Lochgilphead, Argyll. (Vice-Chairman, Educational Committee, since 1927.)

1923. Ross, Thomas Arthur, M.D., C.M., F.R.C.P.Edin., M.R.C.P.Lond., Medical Director, Cassel Hospital, Swaylands, Penshurst, Kent.

1899. Rotherham, Arthur, M.A., M.B., B.Ch.Camb., Lord Chancellor's Visitor; Elm House, Marshall Road, Farncombe, Surrey.

1922. Roy, John Allen Chisholm, M.B., Ch.B.Vict., Medical Superintendent, Royal Hospital, Cheadle, Cheshire.

1924. Rudolf, Gerald de Montjoie, M.R.C.P.Lond., D.P.H., D.P.M., Medical Superintendent, Brentry Colony; Brentry House, Westbury-on-Trym, Bristol.

1929. Russell, David, M.B., Ch.B.Glasg., 183, Saracen Street, Possilpark, Glasgow, N. 
1923. Russell, John, M.B., Ch.B.Glasg., D.P.M., Assistant Medical Officer, West Riding Mental Hospital, Menston, Leeds.

1912. Russell, John Ivison, M.B., Ch.B., F.R.F.P.S.Glasg., D.P.M., M.P.C., Medical Superintendent, North Riding Mental Hospital, Clifton, York. (Secretary, N. and $M$. Division, since 1929.)

1915. Russell, William, M.C., M.D., Ch.B.Edin., Dip. Psych., D.T.M., Commissioner for Mental Hygiene, Union Buildings, Pretoria, South Africa.

1912. Rutherford, Cecil, B.A., M.B., B.Ch.Dubl., Assistant Medical Officer, Holloway Sanatorium, Virginia Water, Surrey.

1907. Rutherford, Henry Richard Charles, F.R.C.S., L.R.C.P.Irel., D.P.H., Medical Superintendent, Farnham House, Finglas, co. Dublin.

1896. Rutherford, James Mair, M.B., C.M., F.R.C.P.Edin., M.P.C., Brislington House, Bristol.

1902. Sall, Ernest Frederick, M.R.C.S., L.R.C.P.Lond., Medical Superintendent, City Mental Hospital, Canterbury.

1908. Samuels, William Frederick, L..M., L.Ch.Dubl., Kearsney Court, near Dover.

1932. Sanders, Marjorie Elizabeth Frances, M.B., Ch.B.Edin., D.P.M., Assistant Medical Officer, Long Grove Mental Hospital, Epsom.

1923. Sang, Janet Adeline Agnes, L.R.C.P.\&S.Edin., L.R.F.P.S.Glasg., Long Acre, Station Road, Scholes, Leeds.

I894. Sankey, Edward Hugh Octavius, M.A., M.B., B.Ch.Camb., Resident Medical Licensee, Boreatton Park, Baschurch, Salop.

1932. Schneider, Frank Edwin Ernest, M.D.Lond,, M.R.C.S., L.R.C.P., D.P.M., Medica Superintendent, Rampton State Institution for Mental Defectives, Retford, Notts.

1928. Scholberg, Harold Alfred, M.B., M.R.C.S., L.R.C.P.Lond., D.P.H., Pathologist, Cardiff City Mental Hospital ; 3, St. Andrew's Crescent, Cardiff.

1926. Scoresby-Jackson, Margaret, M.D., B.S.Durh., c/o Union Bank of Scotland, Ltd., 62, Cornhill, E.C. 3.

1925. Scott, Francis Leonard, M.R.C.S., L.R.C.P.Lond., D.P.M., Deputy Medical Superintendent, Bracebridge Mental Hospital, Lincoln.

1930. Scott, James, M.B., B.Ch., Assistant Medical Officer, District Mental Hospital, Carlow, Ireland.

I9II. Scroope, Gervace Wm. Mavy, M.B., B.Ch.Dubl., Assistant Medical Officer, Central Criminal Asylum, Dundrum, co. Dublin.

I880. Seccombe, George Samuel, M.R.C.S., L.R.C.P.Lond., c/o Lloyds Bank, Threadneedle Street, London, E.C. 3 .

1925. Selkirk, Elizabeth Thompson, M.B., Ch.B.Edin., Deputy Medical Superintendent, Hollymoor Mental Hospital, Northfield, Birmingham.

1929. Selling, Lowell Sinn, B.A.Mich., M.A.Columbia, D.N.B., Sc.M.New York, M.D.Bellevue : Institute for Juvenile Research, 907, South Lincoln Street, Chicago, IU., U.S.A

1912. Sergeant, John Noel, M.B., B.S., M.R.C.S., L.R.C.P.Lond., Medical Superintendent, Newlands House, Tooting Bec Common, London, S.W. I7. (Secretary, South. Eastern Division, since I9I3.)

1925. Shand, George Ernest, M.D., Ch.B.Aberd., D.P.H., 6, Waverley Terrace, St. Saviour's, Jersey.

1930. Sharpe, John Smith, M.B., Ch.B.Glasg., Assistant Medical Officer (Biochemist), County Mental Hospital, Stafford.

rgor. Shaw, Benjamin Henry, M.D., B.Ch.R.U.I., Medical Superintendent, County Mental Hospital, Stafford. (Chairman, N. and M. Division, since 1932; Secretary, Research and Clinical Committee, r927-31.)

1905. Shaw, Charles John, M.D., Ch.B., M.R.C.P.Edin., J.P., Medical Superintendent, Royal Asylum, Montrose.

1904. Shaw, Patrick, L.R.C.P.\&S.Edin., L.R.F.P.S.Glasg., Medical Superintendent, Hospital for Insane, Ballarat, Victoria, Australia.

1909. Shaw, William Samuel Jagoe, M.D.Belf., M.B., B.Ch.R.U.I., Lt.-Col. I.M.S. (ret.), Calcote House, Sandicotes Road, Parkstone, Dorset.

1920. Shearer, Christina Hamilton, M.B., Ch.B.Glasg., Senior Medical Officer, Cassel Hospital, Swaylands, Penshurst, Kent.

1928. Shepherd, Andrew, M.B., Ch.B.Glasg., D.P.M., Assistant Medical Officer, Hill End Mental Hospital, St. Albans, Herts.

1923. Shepherd, Charles Ernest Alan, M.R.C.S., L.R.C.P.Lond., D.P.M., Senior Assistant Medical Officer, Hants County Mental Hospital, Knowle, Fareham. 
1927. Shera, Arthur Geoffrey, M.A., M.D., B.Ch.Camb., M.R.C.S., L.R.C.P.Lond., Pathologist, Eastbourne Hospitals and East Sussex County Mental Hospital, Hellingly; ro, Upperton Gardens, Eastbourne.

1928. Sheridan, Alfred, L.R.C.P.\&S.Irel., Resident Medical Superintendent, Mayo County Mental Hospital, Castlebar, Ireland.

1914. Sherlock, Edward Birchall, B.Sc., M.D.Lond., D.P.H., Barrister-at-Law, " Windleshaw " Whiteleaf Road, Princes Risborough, Bucks.

1914. Shield, Hubert, M.C., M.B., B.S.Durh., c/o Glyn, Mills \& Co., 3, Whitehall Place, S.W. I.

1929. Shilvock, William Henry, B.Sc., M.B., Ch.B.Birm., Assistant Medical Officer, Rubery Hill Mental Hospital, Birmingham.

192 3. Shore, George William, M.D.Lond., D.P.H., D.P.M., Assistant Medical Officer, Springfield Mental Hospital, near Tooting, London, S.W. I7.

1922. Shortt, Jane Elder, M.B., Ch.B.Glasg., Valleyfields, Alingdon, Lanarkshire.

1928. Silverston, Joseph Denzil, M.B., D.S.Durh., Deputy Medical Superintendent, County Mental Hospital, Lançaster.

1928. Sinclair, Arthur Crawford, M.D.Belf., D.P.M., Assistant Medical Officer, Essex County Mental Hospital, Brentwood.

1921. Skene, Leslie Henderson, M.C., M.B., Ch.B.Edin., Dipl. Psych., Medical Superintendent, Mental Hospital, Union Mills, Isle of Man.

1925. Skottowe, James Stewart Ian, M.D.Glasg., D.P.M., Medical Superintendent, Swansea and Merthyr Tydfil Mental Hospital, Sketty, Swansea.

1914. Slaney, Chas. Newnham, M.R.C.S., L.R.C.P.Lond., Medical Officer, H.M. Prison, Wandsworth, S.W. 17; 17, Heathfield Road, Wandsworth Common, London, S.W. 18.

1901. Slater, George Nathan Oscroft, M.D., M.R.C.S., L.R.C.P.Lond., D.P.M., St. Mary's, New Buckenham, Norfolk.

1928. Smith, Arthur Wallace Herbert, M.R.C.S., L.R.C.P.Lond., D.P.M., Assistant Medical Superintendent, Central Mental Hospital, Tanjong Rambutan, Perak, Federated Malay States.

1910. Smith, Gayton Warwick, M.D.Lond., B.S.Durh., M.R.C.S., L.R.C.P.Lond., D.P.H., Senior Assistant Medical Officer, Springfield Mental Hospital, near Tooting, London, S.W. 17.

1905. Smith, George William, O.B.E., M.B., Ch.B.Edin., Wyke House, Isleworth, Middlesex. (Treasurer since 1931.)

1926. Smith, Gordon John, M.B., Ch.B.Aberd., 274, Iffley Road, Oxford.

1923. Smith, Herbert, M.R.C.S., L.R.C.P.Lond., Deputy Medical Superintendent, City Mental Hospital, Fishponds, Bristol.

1899. Smith, John Grimmond, M.D., CM..Edin., "Strathmore", Bowleaze Cove, Weymouth.

1920. Smith, Maurice Hamblin, M.A.Camb., M.D.Durh., M.R.C.S., L.R.C.P.Lond., Medical Officer, H.M. Prison, Birmingham. (Lect. on Crim., Bethlem Royal Hosp.) (CoEditor of Journal since 1931.)

1931. Smith, Robert Sydney Steele, L.M.S.S.A., Assistant Medical Officer, Caterham Mental Hospital, Caterham, Surrey.

191 3. Smith, Thomas Cyril, M.B., Ch.B.Edin., Deputy Medical Superintendent, County Mental Hospital, Barnwood, Gloucester.

1921. Smyth, John Francis, M.D., C.Bh.N.U.I., D.P.M., Inverclyde, Milnsbridge, Huddersfield.

1926. Snell, Harvie Kennard, M.D., B.S., M.R.C.S., L.R.C.P.Lond., D.P.H., Medical Officer, H.M. Prison, Parkhurst ; Camp Hill, Newport, Isle of Wight.

1923. Somerville, George, M.D., Ch.B.Edin., D.P.M., Senior Assistant Medical Officer, West Ham Mental Hospital, Goodmayes, llford.

191 3. Somerville, Henry, B.Sc., M.R.C.S., L.R.C.P.Lond., F.C.S., Harrold, Bedford.

1906. Spark, Percy Charles, M.R.C.S., L.R.C.P.Lond., 3, Clapham Mansions, Nightingale Lane, London, S.W. 4 .

1925. Speer, James Millar Craig, M.B., B.Ch.Belf., Assistant Medical Officer, Wilts County Mental Hospital, Devizes.

1929. Spence, David Sheridan, B.A., M.B., B.Ch., B.A.O.Dubl., Assistant Medical Officer, Hereford County Mental Hospital, Burghill.

1922. Spence, Thomas Reginald Carwardine, M.C., M.B., Ch.B.Edin., Dipl. Psych., Assistant Physician, Royal Edinburgh Hospital for Mental Disorders, Morningside ; 15I, Morningside Drive, Edinburgh.

1901. Starkey, William, M.B., B.Ch.R.U.I., Bryn-y-Neuadd, Llanfairfechan, N. Wales. (Secretary, South-Western Division, 1922-30.) 
1928. Statham, Hugh, M.B., B.C.Camb., M.R.C.S., L.R.C.P.Lond., Physician for Nervous Diseases, Royal Victoria and West Hants Hospital ; 3, Elgin Road, Bournemouth.

1927. Steel, John P., M.D., Ch.B.Edin., Medical Superintendent, Smithdown Road Hospital, Liverpool.

1925. Steel, Samuel Maxwell, M.B., Ch.B.Glasg., Assistant Medical Superintendent, Monyhull Colony, Kings Heath, Birmingham.

r907. Steele, Patrick, M.D., Ch.B., F.R.C.P.Edin., Medical Superintendent, Roxburgh District Mental Hospital; The Hermitage, Melrose.

1929. Stenhouse, Jack Fingland Martin, M.B., Ch.B.Glasg., D.P.M., Assistant Medical Officer, Banstead Mental Hospital, Sutton, Surrey.

1929. Stephen, Adrian Leslie, B.A., M.B., B.S.Lond., Barrister-at-Law, Physician, London Clinic of Psycho-Analysis; I40, Harley Street, W. I.

1914. Stephens, Harold Freize, M.R.C.S., L.R.C.P.Lond., Medical Superintendent, Birmingham Certified Institution, Coleshill Hall, Coleshill, near Birmingham.

1909. Steward, Sidney John, D.S.O., M.D., B.Ch.Camb., M.R.C.S., L.R.C.P.Lond., D.P.H., Dapdune, Guildford, Surrev.

1922. Stewart, Francis Hugh, M.A., D.Sc.St.And., M.D., Ch.B.Edin., D.P.M., Major I.M.S. (retired), Giza Memorial Laboratory, Cairo, Egypt.

1927. Stewart, Francis Melville, M.B., Ch.B.Edin., Deputy Medical Superintendent, County and City Mental Hospital, Littlemore, Oxford.

1931. Stewart, Ronald, M.B., Ch.B.Glasg., Deputy Medical Officer of Health for Mental Services, Corporation of Glasgow : 23, Montrose Street, Glasgow.

I887. Stewart, Rothsay Charles, L.S.A., M.R.C.S.Eng., "The Elms ", Oak End Waye, Gerrard's Cross, Bucks.

I914. Stewart, Roy Mackenzie, M.D., Ch.B., M.R.C.P.Edin., D.P.M., Medical Superintendent, Leavesden Mental Hospital; Coles Farm, Leavesden, Watford.

1905. Stilwell, Henry Francis, L.R.C.P.\&S.Edin., L.R.F.P.S.Glasg., Hayes Park, Hayes End, Middlesex.

I899. Stilwell, Reginald John, M.R.C.S., L.R.C.P.Lond., Moorcroft House, Hillingdon, Middlesex.

1897. Stoddart, William Henry Butter, M.D., B.S., F.R.C.P.Lond., M.R.C.S.Eng., M.P.C. Harcourt House, Cavendish Square, London, W. 1. (Secretary, Educational Committee, 1908-1912.)

1909. Stokes, Frederick Ernest, M.D., Ch.B.Glasg., D.P.H., Senior Assistant Medical Officer, City Mental Hospital, Milton, Portsmouth.

1903. Stratton, Percy Haughton, M.R.C.S., L.R.C.P.Lond,, York Lodge, Cliff Cottage Road, Bournemouth.

1928. Strecker, Herbert A. J., M.D., M.S., Priv.-Doz. Ment. Dis. Univ. Würzb. ; Hon. Research Appointment to the Joint Board of Research for Mental Diseases, City and University of Birmingham; Hollymoor Mental Hospital, Northfield, Birmingham.

1885. Street, Charles Tidbury, M.R.C.S., L.R.C.P.Lond., Haydock Lodge, Newton-le-Willows, Lancs.

1929. Ström-Olsen, Rolf, M.B., B.Ch.Wales, B.Sc., M.R.C.S., L.R.C.P.Lond., D.P.M., Medical Assistant, Cardiff City Mental Hospital, Whitchurch.

1909. Stuart, Frederick Joshua, O.B.E., M.R.C.S., L.R.C.P.Lond., Medical Superintendent, Northampton County Mental Hospital, Berry Wood

1927. Stungo, Ellis, L.R.C.P.\&S.Edin., L.R.F.P.S.Glasg., I6, Craven Park, Harlesden, London, N.W. 10.

1924. Sturrock, Alexander Corsar, M.A., M.D., C.M.Edin., M.R.C.P.Lond., Preston House, Eccles, Manchester. (Physician, Salford Royal Hospital.)

190o. Sturrock, James Prain, M.A.St.And., M.D., C.M.Edin., Commissioner, General Board of Control for Scotland, 25, Palmerston Place, Edinburgh.

1886. Suffern, Alex. Canning, O.B.E., M.D., M.Ch.R.U.I., Glan-y-Mor, Hill Head, Fareham.

1921. Suffern, Canning, M.A., M.B., B.Ch.Camb., M.R.C.S., L.R.C.P.Lond., Glan-y-Mor, Hill Head, Fareham.

1922. Sullivan, Patrick Daniel, F.R.C.S., L.R.C.P.Irel., Medical Superintendent, Verville Asylum, Clontarf, co. Dublin ; 44, Harrington Street, Dublin.

1918. Sutherland, Francis, M.B., Ch.B.Edin., D.P.H., Public Health Department, Dingwall, Ross-shire.

1919. Suttie, Ian D., M.B., Ch.B., F.R.F.P.S.Glasg., I, Taviton Street, London, W.C. r.

1928. Sykes, Elizabeth Sarah Marples, M.B., Ch.B.Sheff., Assistant Medical Officer, South Yorks Mental Hospital, Wadsley, Sheffield. 
1928. Sykes, Kathleen Annie Harvey, M.D., B.S.Lond., M.B., Ch.B.Liverp., D.P.M., Assistant Medical Officer, Rubery Hill Mental Hospital, Birmingham.

1908. Swift, Eric W. D., M.B.Lond., Physician-Superintendent, Valkenberg Mental Hospital, Observatory, Cape Town, 'South Africa.

1926. Talbot, Geoffrey, B.Sc., M.B., Ch.B.Manch., Assistant Medical Officer, Lancashire County Mental Hospital, Prestwich, Manchester.

1923. Tattersall, Stanley Roy, M.R.C.S., L.R.C.P.Lond., Deputy Medical Superintendent, County Mental Hospital, Prestwich, Manchester.

193I. Taylor, Arthur Leslie, M.D.Leeds, Ch.B., Hon. Pathologist, Bristol General Hospital ; Visiting Pathologist, Bristol Mental Hospital ; 5, Rodney Place, Clifton, Bristol.

1910. Taylor, Arthur Loudoun, B.Sc., M.B., Ch.B., F.R.C.P.Edin., Senior Assistant Medical Officer, Stafford County Mental Hospital, Burntwood, Lichfield.

1924. Taylor, Frederic Cecil Marsh, M.R.C.S., L.R.C.P.Lond., D.P.M., Assistant Medical Officer, Kent County Mental Hospital, Chartham Downs.

1897. Taylor, Frederick Ryott Percival, M.D., B.S., M.R.C.S., L.R.C.P.Lond., Medical Superintendent, East Sussex County Mental Hospital, Hellingly. (Chairman, Educational Committee, since 1927; Chairman, South-Eastern Division, 1930-32.)

1932. Taylor, Richard Hamilton, M.B., B.Ch., B.A.O., D.P.H.N.U.I., Pathologist, Grangegorman Mental Hospital, Dublin; 35, Lower Baggot Street, Dublin.

1925. Taylor, Robert, L.R.C.P.\&S.Irel., Assistant Medical Officer, St. Patrick's Hospital, Dublin.

1926. Tennent, Thomas, M.B., Ch.B.Glasg., D.P.H., D.P.M., Deputy Medical Superintendent, Maudsley Hospital, Denmark Hill, London, S.E. 5.

1921. Thomas, Cyril James, M.R.C.S., L.R.C.P.Lond., D.P.M., Deputy Medical Superintendent, Lancashire County Mental Hospital, Whittingham, near Preston.

1920. Thomas, Frederic Percival Selwyn, M.D., Ch.B.Vict., The Oaks, Porthill, Staffordshire.

1908. Thomas, Joseph David, B.A., M.B., B.C.Camb., "Dan-y-Graig", Cheyne Road, Stoke Bishop, Bristol.

rgrr. Thomas, William Rees, M.D., B.S., M.R.C.S., M.R.C.P.Lond., D.P.M., M.P.C., Senior Commissioner, Board of Control; 34, Chartfield Avenue, Putney Hill, London, S.W. 15 .

1925. Thompson, Robert, M.B., B.Ch.Belf., D.P.M., Senior Assistant Medical Officer, St. Patrick's Hospital, Dublin. (Secretary, Irish Division, since 1929.)

1921. Thomson, Aidan Gordon Wemyss, M.B., Ch.B.Glasg., D.Psych., Assistant Physician, Glasgow Royal Mental Hospital, Gartnavel.

1920. Thomson, William George, M.A., M.B., Ch.B.Aberd., D.P.H., D.P.M., Deputy Medical Superintendent, Royal Hospital, Cheadle, Cheshire.

1927. Thorpe, Frederick Thomas, M.R.C.S., L.R.C.P.Lond., D.P.M., Assistant Medical Officer and Pathologist, South Yorkshire Mental Hospital, Wadsley, Sheffield.

1914. Tisdall, Charles Jerome, M.B., Ch.B.Edin., Medical Superintendent, Boughton Hall, Chester.

1903. Topham, J. Arthur, B.A.Camb., M.R.C.S., L.R.C.P.Lond., 7, Montacue Court, Westbourne Gardens, Folkestone.

1896. Townsend, Arthur Allen Deykin, M.D., B.Ch.Birm., M.R.C.S., L.R.C.P.Lond., Medical Superintendent, Barnwood House, Gloucester.

1903. Tredgold, Alfred Frank, M.D.Durh., F.R.C.P.Lond., F.R.S.Edin., "St. Martin's," Guildford. (Lect. on Ment. Deficiency, London Univ.)

1908. Tuach-Mackenzie, William, M.D., Ch.B.Aberd., Physician-Superintendent, Royal and District Asylums, Dundee ; Westgreen, Dundee. (Lect. on Ment. Dis., Univ. of St. Andrews.)

1906. Turnbull, Peter Mortimer, M.C., M.B., B.Ch.Aberd., D.P.M., Medical Superintendent, Tooting Bec Mental Hospital, London, S.W. I7.

1909. Turnbull, Robert Cyril, M.D., M.R.C.S., L.R.C.P.Lond., Medical Superintendent, Essex County Mental Hospital, Severalls, Colchester.

1906. Turner, Frank Douglas, M.B., M.R.C.S., L.R.C.P.Lond., Medical Superintendent, Royal Eastern Counties Institution, Colchester. (PREsident-Elect, 1933-34.)

1922. Twomev, John Christopher, M.B., Ch.B.Liverp., D.P.H., Senior Assistant Physician, The Mental Hospital, Queenstown, Cape Province, South Africa; c/o Secretary for Interior, Pretoria.

1930. Tyars, Mary Elizabeth, B.Sc., M.B., B.S.Lond., D.P.M., Assistant Medical Officer, Horton Mental Hospital, Epsom, Surrey. 
19 29. Umney, William Francis, M.D., M.R.C.S., L.R.C.P.Lond., Medical Superintendent, The Flower House, Catford, S.E. 6 ; 9, The Knoll, Beckenham, Kent.

1931. Valentine, James, M.B., Ch.B.Glasg., Assistant Physician, Glasgow Royal Mental Hospital ; ro55, Great Western Road, Glasgow.

1922. Viehoff, Herman Crowther, M.R.C.S., L.R.C.P.Lond., 48, Moor Lane, Great Crosby, Lancs.

I 894. Vincent, William James N., C.B.E., M.D., B.S.Durh., M.R.C.S., L.R.C.P.Lond., Medical Superintendent, South Yorkshire Mental Hospital, Wadsley, Sheffield. (Lect. on Ment. Dis., Univ. of Sheff.)

1923. Wadsworth, George Reginald, M.B., B.Ch.Belf., D.P.M., Assistant Medical Officer, Lancashire County Mental Hospital, Lancaster.

1928. Waldo, Henry Cecil, M.R.C.S., L.R.C.P.Lond., Barrister-at-Law, Medical Superintendent, Notts County Mental Hospital, Radcliffe-on-Trent, Notts.

1926. Walk, Alexander, M.D., B.S.Lond., D.P.M., Assistant Medical Officer, Cane Hill Mental Hospital, Coulsdon (Assistant Editor, 1928-31, and Co-Editor of Journal since 1931.)

1914. Walker, Robert Clive, M.D., Ch.B.Edin., Deputy Medical Superintendent, West Riding Mental Hospital, Menston, Leeds.

1908. Wallace, John Andrew Leslie, M.D., Ch.B.Edin., M.P.C., J.P., Mental Hospital, Callan Park, Sydney, New South Wales.

1931. Wallace, May Isabel, M.D., Ch.B.Birm., D.P.M., Assistant Medical Officer, Hanwell Mental Hospital, Southall, Middlesex.

1912. Wallace, Vivian, L.R.C.P.\&S.Irel., D.P.H., Ballinakill, Multyfarnham, co. Westmeath.

1932. Walsh, Fergus O'Connell, L.R.C.P.\&S.Irel., Assistant Medical Officer, Portlaoighise (Maryborough) District Mental Hospital, Portlaoighise, co. Leix, Ireland.

1928. Walsh, Michael Anthony, L.R.C.P.\&S.I., D.P.M., Assistant Medical Officer, Claybury Mental Hospital, Woodford Bridge, Essex.

1889. Warnock, John, C.M.G., B.Sc., M.D., C.M.Edin., M.R.C.S.Eng., The Limes, 18r, London Road, Twickenham.

1927. Waterhouse, Amyas Theodore, M.A., M.D., B.Ch.Oxon., M.R.C.S., L.R.C.P.Lond., Hon. Physician, Neurological Department, Radcliffe Infirmary; 35, Beaumont Street, Oxford.

1895. Waterston, Jane Elizabeth, M.D.Brux., F.R.C.P.Irel., L.R.C.S.Edin., M.P.C., Govt. Official Visitor, Valkenberg Mental Hospital, Cape Town; 85, Parliament Street, Cape Town, South Africa.

1922. Watson, Douglas Chalmers, M.D., F.R.C.P.Edin., Physician, Royal Infirmary ; 28, Melville Street, Edinburgh.

1908. Watson, Hugh Ferguson, M.D., Ch.B.Glasg., Ph.D.Edin., L.R.C.P.\&S.Edin., F.R.F.P.S. Glasg., D.P.H., F.R.S.Edin., Deputy Commissioner, General Board of Control for Scotland ; 25, Palmerston Place, Edinburgh.

1924. Watson, John, M.C., M.B., B.Ch.Edin., Resident Medical Superintendent, District Asylum, Londonderry.

1911. Webber, Leonard Mortis, M.R.C.S., L.R.C.P.Lond., Senior Assistant Medical Officer, Surrey County Mental Hospital, Netherne, Coulsdon.

1929. Weber, Hilda Marion, B.Sc., M.D.Lond., Physician, The Institute of Medical Psychology ; 23, New Cavendish Street, London, W. I.

1922. Webster, William Leckie, M.B., Ch.B.Edin., M.P.C., Major R.A.M.C., Specialist in Mental Diseases, Northern Command, British Military Hospital, Rawalpindi, India.

1932. Weir, Thomas William Harold, M.B., B.Ch., B.A.O.Belf., Assistant Medical Officer, County Mental Hospital, Armagh.

r919. Westrup, Joseph Perceval, M.R.C.S., L.R.C.P.Lond., c/o Lloyds Bank, Ltd., Salisbury.

I9II. White, Edward Barton Cartwright, M.R.C.S., L.R.C.P.Lond., Medical Superintendent, City Mental Hospital, Fishponds, Bristol. (Lect. on Ment. Dis., Univ. of Brist.) (Chairman, S.W. Division, since I930.)

1884. White, Ernest William, C.B.E., M.B., M.R.C.P., “ Fenstanton ", Christchurch Road, S.W. 2; and Betley House, near Shrewsbury. (Emeritus 'Prof. Psychol. Med., King's Coll., Lond.) (First Secretary, S.E. Division, 1897-1900; Chairman, Parliamentary Committee, 1904-7; President, 1903-4.

1921. Whitelaw, William, M.B., B.Ch.Glasg., Oaklands, Shirley Green, Acocks Green, Birmingham. 
1889. Whitwell, James Richard, M.B., C.M.Edin., 66, York Mansions, Battersea Park, S.W. Ir. (Acting Hon. Librarian and Chairman, Library Committee, since 1927 ; Hon. Librarian since 1928.)

191 3. Wilkins, William Douglas, M.B., Ch.B.Vict., M.R.C.S., L.R.C.P.Lond., D.P.M., Senior Assistant Medical Officer, Stafford County Mental Hospital, Cheddleton, Leek.

1900. Wilkinson, Harry Bacon, M.R.C.S., L.R.C.P.Lond., "Dalestorth", Lynton Drive, Hillside, Southport, Lancs.

1929. Will, George Wishart, M.B., Ch.B.N.Z., M.P.C., Major R.A.M.C., British Military Hospital, Deolali, Bombay Presidency, India.

1925. Williams, Edward Lincoln, M.R.C.S., L.R.C.P.Lond, The Hall, Harrow Weald, Middlesex.

1922. Williamson, David Hardie, M.B., Ch.B.Edin., Assistant Medical Officer, Glasgow District Mental Hospital, Woodilee, Lenzie, N.B.

1923. Wilson, Alban, M.R.C.S., L.R.C.P.Lond., D.P.M., Medical Superintendent, Coldeast Colony, Sarisbury, Southampton.

1927. Wilson, Charles Herbert, M.B., B.Ch.Dubl., 52, Fitzwilliam Square, Dublin.

1928. Wilson, Edward Alexander, M.D., Ch.B.Edin., Port Darwin, Falkland Islands.

1925. Wilson, Harriette Appleby, M.B., Ch.B.Leeds, D.P.M., Senior Assistant Medical Officer, West Riding Mental Hospital, Wakefield.

1930. Wilson, Henry Leonard, M.B., B.Ch.Camb., M.R.C.P., D.P.M., Physician, The Institute of Medical Psychology ; I42, Harley Street, London, W. I.

1923. Wilson, Isabel Grace Hood, M.D., Ch.B.Edin., D.P.M., Commissioner, Board of Control ; 154, Clarence Gate Gardens, London, N.W. I.

1920. Wilson, James Leitch, M.B., Ch.B.Edin., D.P.M., ro, New Cavendish Street, London, W. I.

1899. Wolseley-Lewis, Herbert, M.D.Brux., F.R.C.S., L.R.C.P.Lond., " Dormers ", Wimborne, Dorset. (Secretary, Parliamentary Committee, 1907-12; Chairman, 1912-21.)

1921. Wood, Bertram William Francis, M.B., B.S.Leeds, West African Medical Staff; c/o P.O., Lagos, South Province, Nigeria.

1929. Woodcock, Oswald Hampson, M.D.Manch., 22, Ridge Hill, Golder's Green, N.W. Ir.

1912. Woods, James Cowan, B.A.R.U.I., M.D., B.S., M.R.C.S., L.R.C.P.Lond., 40, Harley Street, London, W. x. (Lect. on Ment. Dis., St. George's and London Hosps.)

I885. Woods, John Francis, M.D.Durh., L.S.A., M.R.C.S.Eng., 7, Harley Street, London, W. I.

1912. Wootton, John Charles, M.C., M.R.C.S., L.R.C.P.Lond., Medical Superintendent, Haydock Lodge, Newton-le-Willows, Lancs.

1922. Wootton, Leonard Henry, M.C., B.Sc., M.B., B.S., M.R.C.S., L.R.C.P.Lond., D.P.M., Medical Superintendent, Ewell Mental Hospital, Epsom.

1900. Worth, Reginald, O.B.E., M.B., B.S.Durh., M.R.C.S., L.R.C.P.Lond., Medical Superintendent, Springfield Mental Hospital, near Tooting, London, S.W. 17. (Lect. on Ment. Dis., Westm. Hosp.) (Hon. General Secretary since 1919.)

1917. Wright, Maurice Beresford, O.B.E., M.D., C.M.Edin., Physician, The Institute of Medical Psychology ; 86, Brook Street, London, W. I.

r929. Wyllie, Andrew McNae, B.Sc., M.B., Ch.B., Clinical Pathologist, Crichton Royal Institution, Dumfries.

1928. Yates, Arthur Gurney, M.A., M.D.Edin., F.R.C.P.Lond., Physician, Sheffield Royal Infirmary ; 53, Wilkinson Street, Sheffield.

1921. Yellowlees, David, M.B., Ch.B.Glasg., 5, Ruskin Terrace, Glasgow, W. 2.

1914. Yellowlees, Henry, O.B.E., M.D., Ch.B., F.R.C.P.Edin., F.R.F.P.S.Glasg., M.R.C.P. Lond., D.P.M., 93A, Harley Street, London, W. x. (Lect. on Psych. Med., St. Thomas's Hospital, London.)

1926. Young, Hubert Turner Penn, M.B., Ch.B.Edin., Medical Officer, H.M. Prison ; 168, Ducane Road, London, W. 12.

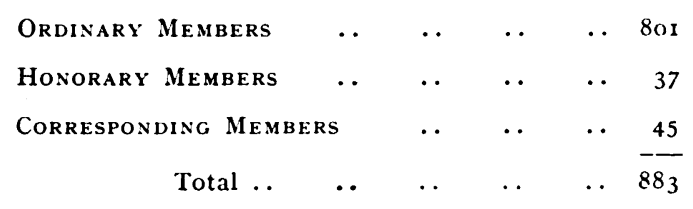




\section{OBITUARY.}

Honorary Members.

1925. Drummond, Sir David, C.B.E., M.A., D.C.L., M.D., J.P., 6, Saville Place, Newcastleon-Tyne.

1927. Robertson, George Matthew, M.D., C.M., F.R.C.P.Edin., Hon. F.R.C.S.Edin., M.P.C., Professor of Psychiatry, University of Edinburgh; Tipperlinn House, Morningside Place, Edinburgh. (Ord. Mem., 1887; PREsIdent, 1922-23; Vice-Chairman, Research and Clinical Committee, since 1927.)

Corresponding Member.

1922. Kure, Prof. Schuzo, Tokyo University, Japan.

Members.

1892. Beadles, Cecil F., M.R.C.S., I.R.C.P.Lond., Gresham House, Egham Hill, Egham, Surrey.

1918. Blandford, Walter Folliott, B.A.Camb., M.R.C.S., L.R.C.P.Lond., 35, Gunterstone Road, W. 14 .

1923. Gibson, George Herbert Rae, D.S.O., M.D., F.R.C.P.Edin., L.C.P.S.Brit. Columbia, Dipl. Psych.; Deputy Commissioner, General Board of Control, Scotland; 23 , Cluny Terrace, Edinburgh.

1912. Hunter, George Yeates Cobb, M.R.C.S., L.R.C.P.Lond., M.P.C., I.M.S. (Ret.), c/o Messrs. Grindlay \& Co., 54, Parliament Street, London, S.W. I.

1905. McDougall, Alan, M.D., Ch.B.Vict., M.R.C.S., L.R.C.P.Lond., Medical Director, The David Lewis Colony, Warford, Alderley Edge, Cheshire.

1903. Nelis, William F., M.D.Durh., L.R.C.P.Edin., L.R.F.P.S.Glasg., The Chalet, New Road, Teignmouth.

I869. Nicolson, David, C.B., LL.D., M.D., C.M.Aberd., M.R.C.P.Edin., Hanley, Park Road, Camberley, Surrey. (PRESIDENT, 1895-6.)

189r. Pierce, Bedford, M.D., F.R.C.P.Lond., "Bankcroft", Douglas Road, Harpenden, Herts. (Secretary, N. and M. Division, 1900-8; PRESIDENT, 1919-20.)

I889. Pope, George Stevens, L.R.C.P.\&S.Edin., L.R.F.P.S.Glasg., Park House, Eaton Road, Norwich.

I89 I. Skeen, James Humphry, M.B., C.M.Aberd., M.P.C., Medical Superintendent, New Saughton Hall, Polton, Midlothian.

I899. Smyth, Walter Samuel, M.B., B.Ch.R.U.I., Assistant Medical Superintendent, District Asylum, Antrim.

1885. Soutar, James Greig, M.B., C.M.Edin., M.P.C., 20, Royal Parade, Cheltenham, (PRESIDENT, 1912-13; Chairman, South-Western Division, 1929-31.) 